\title{
Adjoint-based model predictive control for optimal energy extraction in waked wind farms
}

\author{
Mehdi Vali ${ }^{\mathrm{a}, *}$, Vlaho Petrovića ${ }^{\mathrm{a}}$, Sjoerd Boersma ${ }^{\mathrm{b}}$, Jan-Willem van Wingerden ${ }^{\mathrm{b}}$, Lucy Y. Pao ${ }^{\mathrm{c}}$, Martin Kühn $^{\mathrm{a}}$ \\ ${ }^{a}$ ForWind-Center for Wind Energy Research, University of Oldenburg, Küpkersweg 70, 26129 Oldenburg, Germany \\ ${ }^{b}$ Delft Center of Systems and Control, Delft University of Technology, Mekelweg 2, 2628 CD Delft, The Netherlands \\ ${ }^{c}$ Department of Electrical, Computer \& Energy Engineering, University of Colorado Boulder, USA
}

\begin{abstract}
In this paper, a model predictive control (MPC) is proposed for wind farms to minimize wake-induced power losses. A constrained optimization problem is formulated to maximize the total power production of a wind farm. The developed controller employs a two-dimensional dynamic wind farm model to predict wake interactions in advance. An adjoint approach as an efficient tool is utilized to compute the gradient of the performance index for such a large-scale system. The wind turbine axial induction factors are considered as the control inputs to influence the overall performance by taking the wake interactions into account. A layout of a $2 \times 3$ wind farm is considered in this study. The parameterization of the controller is discussed in detail for a practical optimal energy extraction. The performance of the adjoint-based model predictive control (AMPC) is investigated with time-varying changes in wind direction. The simulation results show the effectiveness of the proposed approach. The computational complexity of the developed AMPC is also outlined with respect to the real time control implementation.
\end{abstract}

Keywords: Nonlinear model predictive control, Adjoint approach, Wind farm control, Wake interactions, Maximal energy extraction

\section{Introduction}

Control of turbines in a wind farm is challenging because of the aerodynamic interactions via wakes. The characteristics of a wake are reduced wind speed and increased turbulence. The former diminishes the total power production of the farm and the latter leads to a higher dynamic loading on the downstream turbines. In a wind farm, wakes often merge wind direction, the surface roughness, turbulence, different atmospheric stratifications, local terrain, and turbine layout in a wind farm [1]. Traditionally each turbine operates greedy, i.e., without optimization at wind farm level. By changing operating points of upwind turbines, it is possible to influence their wakes, and thus the performance of downwind turbines, possibly increasing overall power capture or decreasing structural fatigue loads. Therefore, wind farm control has recently received significant attention in an attempt to lower the levelized cost of energy [2, 3].

The idea to maximize the power production of wind farms in the presence of wakes is to coordinate the control settings of the individual wind turbines, by taking their wake interactions into account. Two commonly proposed but not practically applied approaches for wake control of wind farms are induction control and wake-steering control. In the first method, the upstream wind turbines reduce their own power productions to increase the amount of available kinetic

\footnotetext{
* Corresponding author

Email address: mehdi.vali@uni-oldenburg. de (Mehdi Vali)
} 

yawed out of the wind direction in order to deflect their own wakes away from their downwind turbines [5].

All wind farm control concepts have to be ultimately validated in field experiments, which is a costly and timeconsuming process. Recent field campaigns have demonstrated possibilities for wind farm controllers based on wake deflection [6], as well as the uncertainties stemming from standard wind turbine equipment [7]. Although they cannot fully replace the field experiments, wind tunnel experiments with scaled wind turbine models represent a less time-consuming validation technique characterised by controllable flow conditions, lower expenses and less safety issues. These features were used e.g., in [8] to validate the potential of the torque, pitch and yaw control strategies for wind farm power maximization. However, experimental activities are always limited by the used sensors, and cannot provide a complete information of the flow or wind turbine behaviour. To obtain a more comprehensive understanding of the underlying physics, and how control techniques can influence the flow, numerical simulations, or more specifically, computational fluid dynamics (CFD) models tuned with experimental data, have been widely employed. These models allow resolving time-varying turbulent flow for better characterization of the wake physics, e.g., wake meandering, wake-added turbulence, shape of the velocity deficit inside the wake, multiple wakes, etc. The impacts of different atmospheric stabilities on a deflected wake using yaw control of an upwind turbine have been recently investigated in [9].

Several studies have utilized optimization techniques to find optimal control set-points for the total wind farm performance [10, 11]. Nonetheless, from a control design perspective, these approaches have been either open-loop or quasi steady-state optimization ones, based on analytical static wake models which do not hold fully for dynamical wake interactions. The inherent modeling uncertainties and time-varying inflow conditions, e.g., turbulent nature of wind speed, wind direction changes, and wake meandering demand a closed-loop control approach to react fast enough to the variations of the wake interactions within a wind farm. Extremum-seeking based wind farm control as a closed-loop implementation of the real-time optimization has been proposed for both the induction control [12] and the yaw-based wake steering control [13]. Although this approach benefits from a simple control structure, straightforward for real-time implementation, it suffers from slow convergence time and sensitivity to inevitable uncertainties because of the model-free nature of the control architecture.

Soleimanzadeh et al. [14] have developed a linear state-space model and a distributed controller for the wind farm, which is only valid for small deviations from the equilibrium. Goit and Meyers [15] have proposed an optimal control of energy extraction, utilizing a full 3D Large-Eddy Simulation (LES) model and a receding horizon control approach to increase the turbulent kinetic energy of the flow within a wind farm. The proposed controller relies on a full high-fidelity LES model of the wind farm to compute the optimal control commands, which is too time-consuming for real-time control. LES models of wind farms are vastly expensive, which are typically used for investigation of detailed physics of turbulent flows, wakes and wind turbine interactions with atmospheric boundary layers. Ideally, a control-oriented model should capture relevant dominant flow dynamics in a computationally inexpensive manner, which is the main motivation of studying medium-fidelity dynamic models of wind farms [16, 17, 18].

Vali et al. [19, 20] have proposed an adjoint-based model predictive control (AMPC), which is a closed-loop optimal wind farm control approach, with the main goal of minimizing wake-induced power losses of a wind farm. Figure 1 depicts schematically the control architecture of the proposed optimal control framework. It relies on a two-dimensional dynamic wind farm model, the so-called WFSim [18]. A constrained optimization problem is formulated according to the control objectives and the practical constraints. An adjoint approach is exploited and developed as a cost-effective tool to 
compute the gradient of the performance index. In [21], the AMPC has been investigated to provide active power control (APC) services against undesired wake effects on wind farm responses to grid requirements. In that paper, the AMPC has exploited the non-unique solution of APC for optimal power/load distribution among the individual wind turbines, while their total power production follows a power reference.

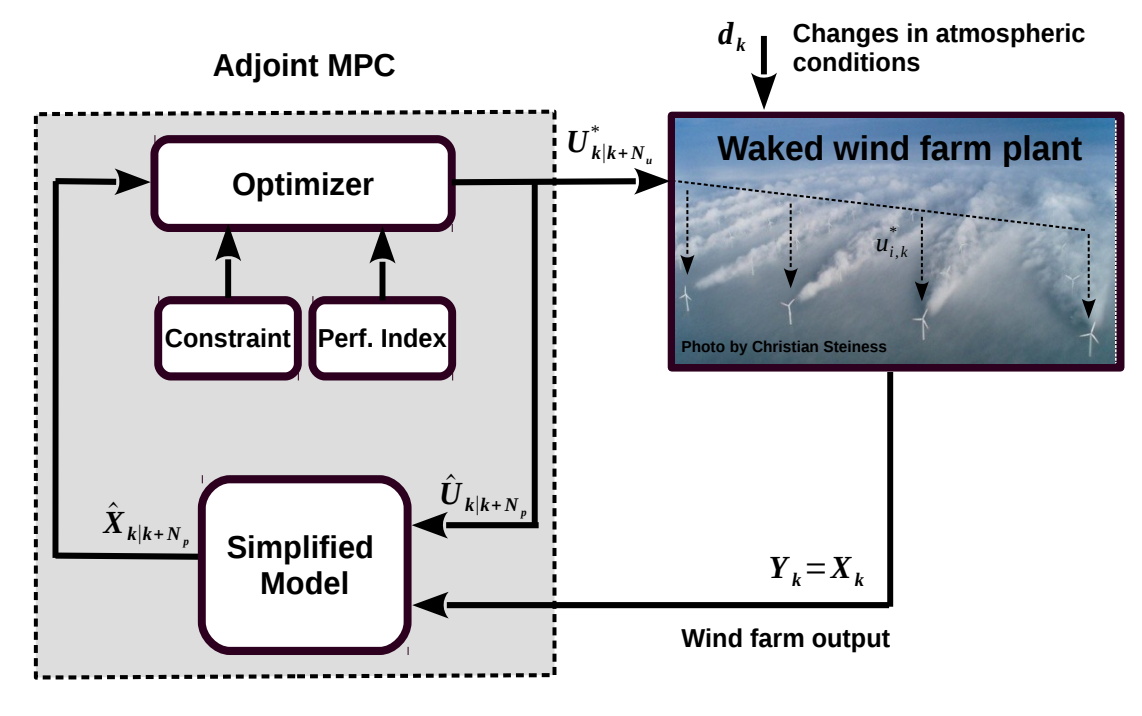

Figure 1: Schematic illustration of the closed-loop optimal control of wind farms. The grey block contains the main components of the adjoint-based model predictive control (AMPC).

It is evident from [20, 21] that the AMPC is potentially capable of optimal control of waked wind farms subjected to time-varying changes in atmospheric and operational conditions. However, several open research questions need to be answered for practical assessments of the proposed approach. From a wind engineering perspective, the employed controloriented model should capture the dominant nature of turbulent flows and wakes for reliable input/output predictions of the wind power plant. The wake propagation from an upwind turbine toward its downwind turbine introduces a time delay, needed to be considered in the AMPC optimization problem for an applied wake control. Furthermore, WFSim is a large-scale dynamic system due to the fact that each grid point contains three states and the order of a wind farm model can easily grow depending on the meshing resolution. Several studies have focused so far to improve the computational efficiency of the approach, e.g., the exploitations of sparse structure in the WFSim system matrices [22], sparse banded matrices to reduce further computational efforts [23], and adjoint approach to compute the gradient of the performance index with the same computational cost of the model [20]. However, the computational complexity of the AMPC is still one major issue for real-time closed-loop control, which becomes prohibitive for large wind farms.

The current paper focuses on the extension and analysis of the AMPC from both the applied control and the wind engineering perspectives. The APMC is developed and investigated here for optimal energy extraction in waked wind farms. The wake propagation time is analyzed as a key information for selecting the AMPC design parameters in order to ensure a satisfactory closed-loop performance and computational efficiency simultaneously. Furthermore, the computational expenses of the adjoint approach are determined using the orders of the model and the optimization domain, which are effectively used to limit the computational burden of the AMPC. The closed-loop performance of the computationally efficient AMPC is evaluated with fast dynamic wake interactions, caused by time-varying changes in wind directions as disturbances. To ensure the applicability of the results and discussions, the employed 2D wind farm model 
is also validated with the PArallelized Large-eddy simulation Model (PALM) of wind farms, which has extensively been used for analyzing the performance of real wind farms under turbulent conditions and their interactions with boundary layers [7, 24].

The remainder of this paper is organized as follows. Section 2 presents briefly the fundamentals of the medium-fidelity model of wind farms. The main focus of section 3 is on the structure of the proposed and the extended version of the AMPC for power maximization of wind farms, consisting of the optimal control problem, the adjoint-based gradient, the optimization method, and the applied practical constraints on the wind turbine control inputs. The investigated wind farm example is introduced in section 4. A comparative study is conducted in order to validate the utilized model with an LES model of wind farms. Then, the design characteristics and the performance of the proposed AMPC is discussed through simulation studies in section 5 . The focus of section 6 is on the computational complexity of the proposed MPC method for wind farm control. Finally, the strengths and weaknesses of the proposed approach are outlined in section 7 as conclusions of the current study.

\section{Wind farm model}

This section briefly presents the fundamentals of the employed control-oriented wind farm model, the so-called WFSim [18, 22, 25]. The model solves the longitudinal and lateral flow velocity components in a wind farm at the wind turbine hub height. The flow velocity components are then used to model the wind turbines as actuator discs and to estimate the power production of the wind farm.

\subsection{The flow model}

The WFSim inflow is modeled using the incompressible 2D momentum Navier-Stokes equations constrained by the continuity equation [18]:

$$
\begin{aligned}
\rho\left(\frac{\partial u}{\partial t}+\nabla \cdot u \mathbf{u}\right) & =-\frac{\partial p}{\partial x}+\mu \nabla^{2} u+f_{x} \\
\rho\left(\frac{\partial v}{\partial t}+\nabla \cdot v \mathbf{u}\right) & =-\frac{\partial p}{\partial y}+\mu \nabla^{2} v+f_{y} \\
\nabla \cdot \mathbf{u} & =-\frac{\partial v}{\partial y}
\end{aligned}
$$

where $\rho$ represents the air density, $\mu$ is the dynamic viscosity, $p$ is the pressure field and $\mathbf{u}=(u, v)^{T}$ is the velocity vector field at hub height. $\boldsymbol{f}=\left(f_{x}, f_{y}\right)^{T}$ represents the external source terms in the $x$ - and $y$-directions, i.e., streamwise and lateral directions, respectively, employed for modeling the wind turbines and their DoFs, e.g., the axial induction factor and the yaw misalignment. Since the focus of the current study is on induction-based wake control, the yaw DoF, described by a lateral thrust force $f_{y}$ in order to deflect the wake downstream of a wind turbine, is neglected for simplicity.

\subsection{The wind turbine model}

Actuator disc theory is employed to exert a thrust force into the incoming flow and extract a certain amount of energy from the wind. The thrust force and the produced power for a single turbine are expressed as follows [26]:

$$
\begin{aligned}
F_{T} & =\frac{1}{2} \rho A_{d} U_{\infty}^{2} C_{T}(a), & C_{T}(a) & =4 a(1-a), \\
P_{T} & =\frac{1}{2} \rho A_{d} U_{\infty}^{3} C_{P}(a), & C_{P}(a) & =4 a(1-a)^{2},
\end{aligned}
$$


where $U_{\infty}$ is the effective wind speed at a far distance upwind from the rotor disc, $A_{d}$ the swept area of the rotor plane, and $C_{T}$ and $C_{P}$ are the thrust and power coefficients of a wind turbine, respectively, which are functions of the axial induction factor $a$. The practical torque and pitch control inputs of a wind turbine can be related to the axial induction factor $a$, which is the ratio of the reduced wind velocity at the rotor plane to the effective wind speed $U_{\infty}$ [26].

Considering the induction effect of a rotor disc in general as

$$
U_{d}=(1-a) U_{\infty}
$$

enables us to estimate the exerted thrust force using the measurable rotor averaged wind velocity $U_{d}$ at the rotor plane and the axial induction factor $a$. Similar to [19], a more precise approximation of $U_{\infty}$ can be achieved using the correction factor $C_{f}(a)$ for a wind turbine model in WFSim as

$$
\hat{U}_{\infty}=\frac{C_{f}(a) U_{d}}{1-a}
$$

The necessity of the introduced correction factor, which is attributed to the two-dimensional inflow of the WFSim model, is demonstrated in Appendix C The described wind turbine model holds under stationary flow conditions. Thus, the induction factor is extended with a first-order lag with the aerodynamic time constant $13.5 \mathrm{~s}$ as suggested in [27].

\subsection{The WFSim model}

The 2D flow is spatially and temporally discretized over a specified staggered grid following [28]. Therefore, the $i^{\text {th }}$ turbine model is incorporated inside the flow model using the following discretized source term:

$$
\boldsymbol{f}_{x_{i}}=-2 \rho \Delta y_{i} \boldsymbol{U}_{d_{i}}^{2} \beta_{i}, \quad \beta_{i}=\frac{a_{i}}{1-a_{i}}
$$

where $\boldsymbol{f}_{x_{i}}$ and $\boldsymbol{U}_{d_{i}}$ collect the discretized thrust forces and the corrected wind velocities, respectively, at the corresponding grid cells which cover the $i^{\text {th }}$ rotor disc. The width of the grid cells $\Delta y_{i}$ is considered constant in this study. The virtual control variable $\beta_{i}$ is defined to obtain linear expressions of the thrust force and the captured power with respect to the wind turbine control input [22]. Note that $\boldsymbol{f}_{x_{i}}$ is calculated only for the flow component of the effective wind speed $U_{\infty}$ perpendicular to the rotor plane.

Finally, the discrete-time wind farm model with $N_{t}$ wind turbines can be represented in a nonlinear descriptor statespace form as follows [18]

$$
\boldsymbol{E}\left(X_{k}\right) X_{k+1}=\boldsymbol{A} X_{k}+\boldsymbol{B}\left(X_{k}\right) \boldsymbol{\beta}_{k}+b\left(X_{k}\right)
$$

with state variable $X_{k}$, which stacks all the longitudinal and lateral flow velocities $\bar{u}_{k} \in \mathbb{R}^{\left(N_{x}-3\right)\left(N_{y}-2\right) \times 1}, \bar{v}_{k} \in$ $\mathbb{R}^{\left(N_{x}-2\right)\left(N_{y}-3\right) \times 1}$, and scaled pressure components $\bar{p}_{k} \in \mathbb{R}^{\left(N_{x}-2\right)\left(N_{y}-2\right) \times 1}$ in every point of the staggered grid with $N_{x}$ and $N_{y}$ being the number of cells in the $x$ - and $y$-direction, respectively. The vector of control inputs $\boldsymbol{\beta}_{k}$ collects the control variables of all $N_{t}$ turbines within the wind farm, i.e., the virtual axial induction factors 8 .

$$
X_{k}=\left(\begin{array}{c}
\bar{u}_{k} \\
\bar{v}_{k} \\
\bar{p}_{k}
\end{array}\right), \quad \boldsymbol{\beta}_{k}=\left(\begin{array}{c}
\beta_{1, k} \\
\vdots \\
\beta_{N_{t}, k}
\end{array}\right) \in \mathbb{R}^{N_{t} \times 1}
$$



optimally adjust the wake interactions. The whole procedure is repeated with new measurements, providing feedback into the optimization problem, which enables the controller to react to varying atmospheric and operating conditions of a wind farm.

The descriptor matrix $\boldsymbol{E}\left(X_{k}\right)$ contains the diffusion and convection terms of the 2D flow constrained to the continuity equation (3) after spatial discretization. The constant matrix $\boldsymbol{A}$ represents the temporal discretization of the flow depending on the chosen sampling time, and the control distribution matrix $\boldsymbol{B}\left(X_{k}\right)$ represents how the axial induction factors of the individual wind turbines influence the wind farm states. Finally, the vector $b\left(X_{k}\right)$ describes the effect of the defined boundary conditions.

\section{Adjoint-based model predictive control}

An adjoint-based model predictive control (AMPC) framework is proposed here for minimizing the wake-induced power losses of wind farms. Figure 2 demonstrates schematically the main components of the designed nonlinear MPC. The control inputs and wake-induced plant responses are predicted in advance and optimized for a finite time horizon $N_{p}$. The optimization method benefits from an adjoint method as a cost-effective tool to compute the gradient of the specified performance index, subjected to the aforementioned medium-fidelity wind farm model. The nonlinear model and performance index are linearized and stored online over the prediction horizon (see blue arrows in Fig. 2). Then, an adjoint variable is defined to estimate the proper search directions (see red arrows in Fig. 2) for iteratively solving the formulated optimal control problem. Indeed, the adjoint variable associates the variations of the model and the performance index over the prediction horizon $N_{p}$, which is informative for calculating the gradient, as explained in subsection 3.2 Finally, the first part of the optimal solution $N_{u}<N_{p}$ is applied to the plant (see green arrows in Fig. 22 in order to

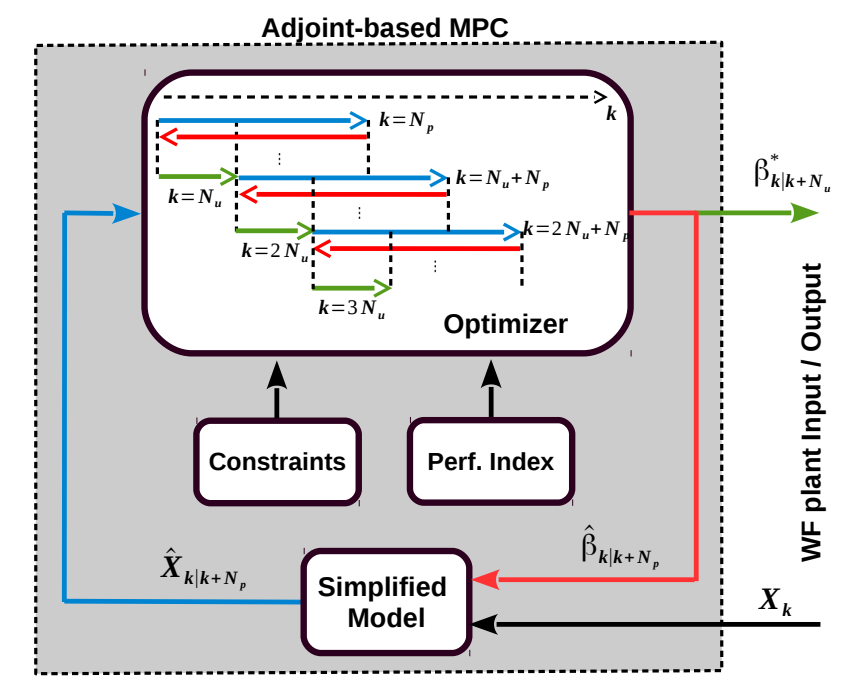

Figure 2: Schematic illustration of the adjoint-based model predictive control (AMPC) of wind farms

Here, it is assumed that all required state variables for optimal control of a given wind farm are measurable. Observation techniques can be used for estimating the state variables of the employed model. Doekemeijer et al. [23, 29] have developed a computationally efficient Ensemble Kalman Filtering (EnKF) method based on WFSim, to estimate the flow velocities at hub height of an LES model of wind farms. It is shown that such estimation techniques are capable of adjusting the predicting model in order to address the model plant mismatches for a proper feedback correction strategy. Moreover, the recent developments of lidar technology make the measurements of the wind speed at various distances 
of wind turbines practical [7] and reliable for developing advanced control algorithms, e.g., model predictive control, in wind energy [30, 31].

Furthermore, the focus of this study is on the centralized structure of a high-level wind farm controller for finding the optimal control set-points of the wind turbines, taking their wake interactions into account. It is assumed that the low-level wind turbine control systems are able to realize the commanded optimal trajectories to correct local mismatches and mitigate short-scale disturbances due to turbulence [32, 33].

\subsection{Optimal control problem}

A constrained optimization problem is formulated here to minimize the wake-induced power losses of a wind farm. The manipulating variables are the virtual wind turbine induction factors $\boldsymbol{\beta}_{\boldsymbol{k}}$ at time instant $k$. The optimal control problem is formulated as finding the maximal power production of the wind farm over a finite time horizon $N_{p}$. Hence, we first define the following performance index, referring to the sum of the actual turbine power productions, at time instant $k$ as

$$
\mathcal{J}_{k}\left(X_{k}, \boldsymbol{\beta}_{\boldsymbol{k}}\right)=\sum_{i=1}^{N_{t}} P_{i, k}
$$

Now, we can formulate the following constrained optimization problem over the prediction horizon $N_{p}$

$$
\begin{array}{ll}
\max _{\tilde{\boldsymbol{\beta}}} & \mathcal{J}(\tilde{X}, \tilde{\boldsymbol{\beta}})=\sum_{k=1}^{N_{p}} \mathcal{J}_{k}\left(X_{k}, \boldsymbol{\beta}_{\boldsymbol{k}}\right), \\
\text { s.t. } & \tilde{\mathbf{C}}(\tilde{X}, \tilde{\boldsymbol{\beta}})=0, \\
& \beta_{i, k}=\beta_{i, k-1} \quad \text { for } \quad k=N_{p}-N_{f}+1, \cdots, N_{p} \\
& 0<\beta_{i, k} \leq \frac{1}{2} \quad \equiv \quad 0<a_{i, k} \leq \frac{1}{3},
\end{array}
$$

The equality constraint (12) represents the discrete-time wind farm model, used for predicting the wake evolutions and interactions over the prediction horizon $N_{p}$ with the following expanded form

$$
\tilde{\mathbf{C}}=\left(\begin{array}{c}
C_{1}\left(X_{0}, X_{1}, \beta_{0}\right) \\
C_{2}\left(X_{1}, X_{2}, \beta_{1}\right) \\
\vdots \\
C_{N_{p}}\left(X_{N_{p}-1}, X_{N_{p}}, \beta_{N_{p}-1}\right)
\end{array}\right), \quad \tilde{X}=\left(\begin{array}{c}
X_{1} \\
X_{2} \\
\vdots \\
X_{N_{p}}
\end{array}\right), \quad \tilde{\boldsymbol{\beta}}=\left(\begin{array}{c}
\boldsymbol{\beta}_{1} \\
\boldsymbol{\beta}_{2} \\
\vdots \\
\boldsymbol{\beta}_{N_{p}}
\end{array}\right),
$$

where according to 9

$$
C_{k}\left(X_{k-1}, X_{k}, \beta_{k-1}\right)=\boldsymbol{E}\left(X_{k-1}\right) X_{k}-\boldsymbol{A} X_{k-1}-\boldsymbol{B}\left(X_{k-1}\right) \boldsymbol{\beta}_{k-1}-b\left(X_{k-1}\right)
$$

The prediction horizon $N_{p}$ should be long enough to capture wake propagations within a wind farm. Therefore, the wake propagation plays a key role in the closed-loop performance, as it introduces a significant time delay to wind farm responses. It is thus common that a short preview objective leads to optimal predictions that yield either an undesired closed-loop performance or even instability issue. The terminal set constraints 13 are introduced to limit the inherent behaviour caused by the finite-horizon control, through blocking the moves over the final time period $N_{f}$, as explained in 
section 3.4 In [20], a long prediction horizon is used to avoid such issues, which demands ineffectively computational expenses. However, we demonstrate here that setting the final time period as $N_{f}=N_{p} / 2$ equals to the wake traveling time from an upwind turbine toward the first downwind turbine would be a fair choice to ensure a desired closed-loop performance and computational efficiencies simultaneously.

The inequality constraint (14) addresses the practical constraints on the wind turbine control inputs. The formulated optimization problem (11)-(14) can be extended for other control objectives of wind farms, e.g., active power control, optimal load coordination among wind turbines [21], and optimal wake steering.

\subsection{Adjoint-based gradient of the cost function}

As mentioned above, the wind farm model given in 9 is a large-scale system. The order of the model is determined by the chosen domain size and staggered grid resolution $\left(N_{x} \times N_{y}\right)$. Although the existing sparsity in the system matrices makes the model computationally efficient, the number of cost function evaluations for each control input using common approaches, e.g., finite difference, make the computation of the gradient intractable and consequently impractical for real-time applications.

Adjoint methods give an efficient way of obtaining the gradient of a performance index including many decision variables, regardless of the system order. Adjoint methods have been used widely in wind energy investigations for wind farm layout optimization [34] and analyses of wind farm control [15, 35] using high-fidelity models of wind farms consisting of a vast number of state variables. Considering the computational efficiency of the WFSim model, we employ an adjoint method here for computing the gradient of the MPC cost function in order to make it practical and realizable for real-time control of wind farms.

First, we define the Lagrangian to turn the constrained optimization problem (11)-12 into an unconstrained one as follows

$$
\mathcal{L}(\tilde{X}, \tilde{\boldsymbol{\beta}}, \Lambda) \equiv \mathcal{J}(\tilde{X}, \tilde{\boldsymbol{\beta}})+\Lambda^{T} \tilde{\mathbf{C}}(\tilde{X}, \tilde{\boldsymbol{\beta}})
$$

where $\Lambda=\left[\lambda_{1}, \lambda_{2}, \ldots, \lambda_{N_{p}}\right]^{T}$ is the vector of Lagrange multipliers. Since the equality constraint $[12$ holds always for $k=1,2, \cdots, N_{p}$, we may choose $\Lambda$ freely, and the gradient of the cost function can be expressed as

$$
\nabla_{\tilde{\boldsymbol{\beta}}} \mathcal{J}=\nabla_{\tilde{\boldsymbol{\beta}}} \mathcal{L}=\left(\mathcal{J}_{\tilde{X}}+\Lambda^{T} \tilde{\mathbf{C}}_{\tilde{X}}\right) \tilde{X}_{\tilde{\boldsymbol{\beta}}}+\mathcal{J}_{\tilde{\boldsymbol{\beta}}}+\Lambda^{T} \tilde{\mathbf{C}}_{\tilde{\boldsymbol{\beta}}}
$$

where $(.)_{\tilde{X}}$ and $(.)_{\tilde{\boldsymbol{\beta}}}$ represent the partial derivatives with respect to $\tilde{X}$ and $\tilde{\boldsymbol{\beta}}$, respectively. Appendix A presents the sparse structure of $\tilde{\mathbf{C}}_{\tilde{X}}$ in A.1 and $\tilde{\mathbf{C}}_{\tilde{\boldsymbol{\beta}}}$ in A.2, describing the perturbations of the employed model in response to the control inputs and disturbances over a prediction interval $N_{p}$.

In order to avoid tedious computation of $\tilde{X}_{\tilde{\boldsymbol{\beta}}}$, one may choose the adjoint variable as a solution of the following adjoint equation [36]:

$$
\tilde{\mathbf{C}}_{\tilde{X}}^{T} \Lambda=-\mathcal{J}_{\tilde{X}}^{T}
$$

The structure of the adjoint equation (18) indicates that the adjoint solution strongly depends on the variations of the performance index and the wind farm model response over the prediction horizon. By substituting the adjoint variable $\Lambda$ into the gradient equation (17), there is no need for tedious calculations of the derivatives of the flow solution with respect 
to the control variables $\left(\tilde{X}_{\tilde{\boldsymbol{\beta}}}\right)$ and the gradient of the performance index can be expressed in the following compact form

$$
\nabla_{\tilde{\boldsymbol{\beta}}} \mathcal{J}=\mathcal{J}_{\tilde{\boldsymbol{\beta}}}+\Lambda^{T} \tilde{\mathbf{C}}_{\tilde{\boldsymbol{\beta}}}
$$

The structure of the matrices $\tilde{\mathbf{C}}_{\tilde{X}}$ in A.1 and $\tilde{\mathbf{C}}_{\tilde{\boldsymbol{\beta}}}$ in $A .2$ allows us to derive the backward time-propagation of the adjoint variable $(18)$ and consequently the gradient $(19)$ as follows

$$
\begin{aligned}
\left(C_{k-1}\right)_{X_{k-1}}^{T} \lambda_{k-1} & =-\mathcal{J}_{X_{k-1}}^{T}-\left(C_{k}\right)_{X_{k-1}}^{T} \lambda_{k} \\
\nabla_{\boldsymbol{\beta}_{k-1}} \mathcal{J} & =\mathcal{J}_{\boldsymbol{\beta}_{k-1}}+\lambda_{k}^{T}\left(C_{k}\right)_{\boldsymbol{\beta}_{k-1}}
\end{aligned}
$$

with initializing $\lambda_{N_{p}+1}=0$ at the end of the prediction interval.

\subsection{Receding horizon control law}

Due to the time-dependent nature of the problem, a descent gradient method is employed to solve the optimization problem iteratively. It is computationally efficient when the number of optimization variables is large due to exploiting few vector operations instead of high-dimensional matrix operations, e.g., in Newton's method [37].

Given an estimated control variable $\tilde{\boldsymbol{\beta}}^{(n)} \in \mathbb{R}^{N_{t} N_{p} \times 1}$ at the $n^{\text {th }}$ optimization iteration, a new estimation is obtained using the computed time-dependent gradient $(19)$ as follows

$$
\tilde{\boldsymbol{\beta}}^{(n+1)}=\tilde{\boldsymbol{\beta}}^{(n)}+\alpha^{(m)}\left(\nabla_{\tilde{\boldsymbol{\beta}}}^{(n)} \mathcal{J}\right)^{T}
$$

with $\alpha^{(m)}$ being the step size along a given search direction, which ensures that an improvement is achieved in the total power production at each optimizing iteration. A backtracking line search method is employed to find the proper step size, initialized with its maximum value $\alpha^{(0)}=1$. Until the improvement in the performance of interest 11 is achieved, the step size at the $m^{\text {th }}$ line search iteration is set as:

$$
\alpha^{(m)}=\frac{1}{2} \alpha^{(m-1)}
$$

The solution $\tilde{\boldsymbol{\beta}}^{\star}$ is obtained when the gradient falls below a user-defined threshold. The receding horizon control law, which is fed to the wind farm is implemented as

$$
\left(\begin{array}{c}
\boldsymbol{\beta}_{1}^{\star} \\
\vdots \\
\boldsymbol{\beta}_{N_{u}}^{\star}
\end{array}\right)=\left[\begin{array}{ll}
\boldsymbol{I}_{N_{u}} & {[\mathbf{0}]_{N_{u} \times\left(N_{p}-N_{u}\right)}}
\end{array}\right] \otimes \boldsymbol{I}_{N_{t}} \tilde{\boldsymbol{\beta}}^{\star},
$$

where $\boldsymbol{I}$ stands for the identity matrix with $N_{u}$ and $N_{t}$ being the length of the receding horizon and the number of wind turbines, respectively, and $\otimes$ represents the Kronecker product. Repeating the whole procedure with new measurements provides feedback into the optimal control problem, which enables the AMPC to react to varying atmospheric and operating conditions. Depending on how fast the wind farm responds to the control inputs and disturbances, the receding horizon control $N_{u}$ determines the quality of the closed-loop performance. Increasing $N_{u}$ degrades the closed-loop performance 
and makes the control system less responsive to fast dynamics, e.g., wake meandering, wind gust, and turbulent flows.

\subsection{Control input constraints}

Without the move-blocking $(13)$, any control actions at the end of the horizon would not be seen by the downwind turbines in (11). Therefore, the optimal solution would be to start using the greedy setting, since the wakes with the lower energy content would not reach downwind turbines within the prediction horizon. Although this undesired behaviour can be avoided in the wind farm closed-loop performance using a long preview prediction, it adds unnecessary computational effort [20]. Therefore, we prevent the AMPC from making such changes by employing the move-blocking (13). Following many practical online MPC systems [38], an input move-blocking scheme [39] is applied for mapping the adjoint-based gradient as follows

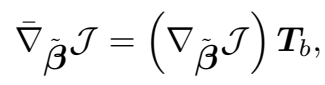

with the blocking matrix

$$
\boldsymbol{T}_{b}=\left[\begin{array}{cc}
\boldsymbol{I}_{N_{p}-N_{f}} & \mathbf{0} \\
\mathbf{0} & \frac{1}{N_{f}}[\mathbf{1}]_{N_{f} \times 1}
\end{array}\right] \otimes \boldsymbol{I}_{N_{t}},
$$

where the Kronecker product $\otimes$ is used to apply the final set constraint $[13]$ on $N_{t}$ wind turbines of the wind farm. In doing so, we eliminate the undesired behaviour caused by the finite-horizon optimization and reduce the computational complexity of the optimal control problem by choosing the prediction horizon $N_{p}$ and the final time period $N_{f}$ according to the specific physics of the problem, e.g., wake traveling time.

An active set method is also employed to enforce the control inputs applied to always remain within the specified limits (14). When the constraint 14 is activated, e.g., $\beta_{i, k}=0.5$ or $\beta_{i, k}=0$, the adjoint-based gradient is projected in order to move along the activated constraint.

\subsection{Computational complexity}

This section establishes the systematic procedure of the AMPC implementation. Figure 3 demonstrates schematically the main components of the developed optimizer for optimal predictive control of wind farms. The grey block contains the main three computation steps of the AMPC as follows

- Step 1, linearization: The employed WFSim model and the performance index are linearized and stored online over the prediction horizon for computing the adjoint variable,

- Step 2, adjoint variable calculation: The adjoint-based gradient is calculated backward in time using the family of linearized models and performance indices with respect to both the state variables and control inputs,

- Step 3, line-search: At least one forward prediction is conducted to ensure that the chosen step size along the search direction yields an improvement in the performance of interest.

The user-defined threshods, e.g. the allowable CPU (central processing unit) time or the desired norm of the gradient, specify the number of optimizing iterations for applying the optimal solution to the wind farm. Therefore, the total elapsed time $T$ for a receding horizon control $\beta_{k \mid k+N_{u}}^{\star}$ can be approximated as

$$
T \approx \sum_{i=1}^{N_{I}}\left(T_{1, i}+T_{2, i}+N_{l} T_{3, i}\right)
$$




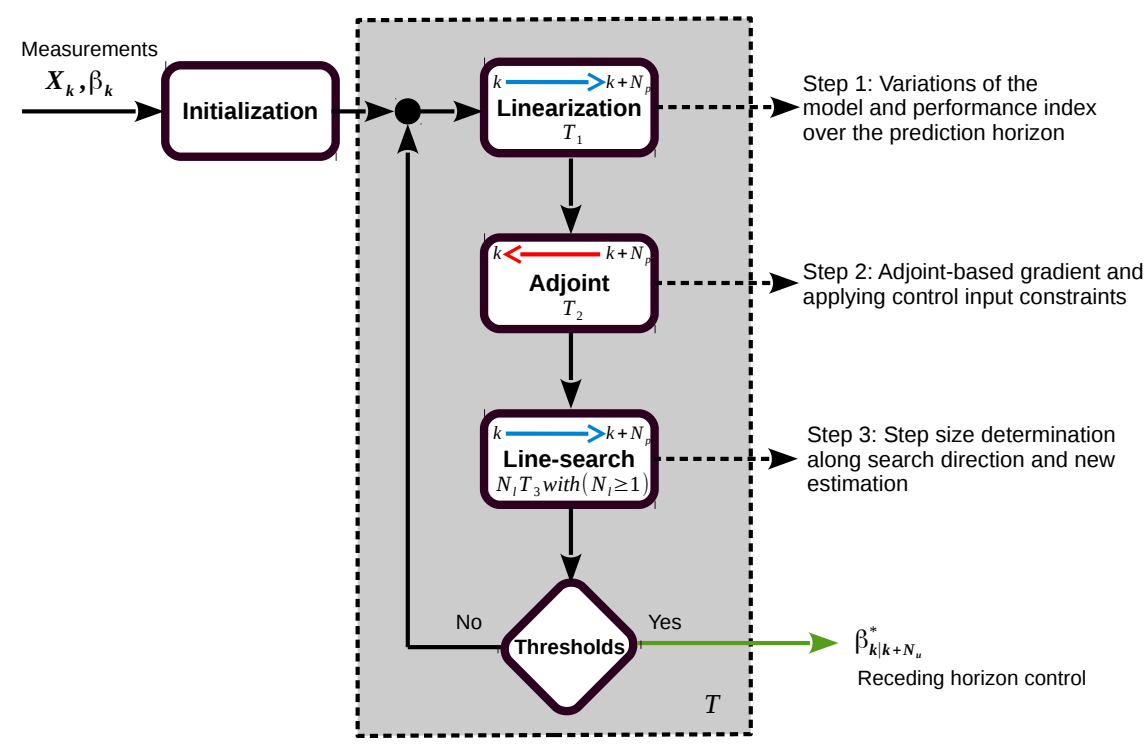

Figure 3: Schematic illustration of the main components of AMPC optimization solver. The grey block contains the main computing steps of AMPC for optimal predictive control of wind farms. $T_{1}, T_{2}$, and $T_{3}$ stand for the elapsed time of each main computing step.

where $N_{I}$ and $N_{l}$ represent the total number of optimization and line-search iterations, respectively and $T_{1, i}, T_{2, i}$, and $T_{3, i}$ stand for the elapsed times of the aforementioned computing step 1 to 3 at $i^{\text {th }}$ iteration. It should be noted that there exist a few scalar and vector operations in between, which their parts are neglected in the total elapsed time $T$, compared with these three main steps.

\section{Model comparison and validation}

For practical perspectives of the approach, we demonstrate that WFSim is able to capture the dominant dynamics of the resolved turbulent wakes of an LES model of wind farms at the hub height of the wind turbines. An LES approach is conceptually suitable for studying the evolution of turbulence and wind turbine wakes because it can simulate a very wide range of turbulent scales. In the current study, we use the PArallelized Large-eddy simulation Model (PALM) [40] coupled with the Actuator Disc Model (ADM) of wind turbines [24]. The PALM model has been used widely for investigating the performance of real wind farms [24] and for analyzing real measurement data [7].

PALM is an open source LES code, which is developed for atmospheric and oceanic flows and optimized for massively parallel computer architectures. It uses central differences to discretize the non-hydrostatic, filtered, and incompressible Boussinesq approximation of the three-dimensional Navier-Stokes equations on a uniformly spaced Cartesian grid [40]. PALM resolves the detailed unsteady turbulent wind farm flow and wind turbine wakes, considering their interactions with the atmospheric boundary layer. On the other hand, the employed WFSim model aims only to estimate the dominant dynamics of wake interactions at the hub height and resultant power losses of downwind turbines, making the model suitable for the wind farm control.

\subsection{Case study}

A layout of a $2 \times 3$ wind farm is considered in the current study (see Fig. 4). The wind turbines with rotor diameter $D=126 \mathrm{~m}$, taken from the freely available model of the NREL 5-MW reference wind turbine [41], are spaced 5D in the stream-wise direction. The rotor centers of the middle turbines are offset half a rotor diameter from the centers of 
the upwind and downwind turbines to create partial wake overlaps with rotors of the downwind turbines. It should be mentioned that analyzing the partial wake overlaps using analytical low-fidelity static wake models, used in many studies such [10, 11], typically yields an unreliable estimation of wind turbine power productions.

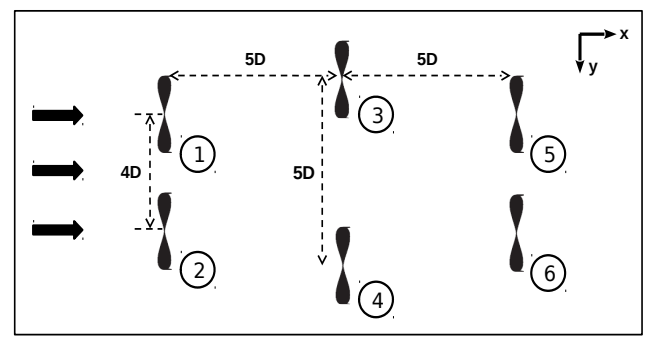

Figure 4: The layout of the investigated $2 \times 3$ wind farm.

Table B.3 summarizes the key parameters of the simulation set-ups and specifications, e.g., the domain size, the grid resolution, the wind turbine control DoF, and atmospheric conditions, for both the studied WFSim and PALM models of the given wind farm example. Furthermore, the characteristic performances of the wind turbine models in both WFSim and PALM are provided in Appendix C, compared with actuator disc theory. For this study, PALM was run on the EDDY HPC (High-Performance Computing) cluster devoted to wind energy research at ForWind-University of Oldenburg [42].

\subsection{Wind farm performance}

A neutral boundary layer (NBL), with a mean wind speed of $8 \mathrm{~m} / \mathrm{s}$ at hub height is simulated in PALM as a reference. Under such conditions, large wake losses can be expected during standard operation of the wind farm. A precursor simulation of the atmospheric boundary layer is conducted without any turbines in order to allow for the generation of a fully developed undisturbed turbulent flow field which can then be used for the initialization of the main simulation run in which a turbulence recycling method is used [40].

One important note is that observations from LES studies [24] and real measurement data [1] of offshore wind farms have shown that there exist different rates of wake recovery behind the wind turbines for neutral boundary layers (NBL), wherein a mixing process with turbulent inflows re-energizes the wake. This fact is also considered in the current validations of the WFSim. A tunable wake recovery model is introduced in [18] based on the spatial parameterization of the dynamic viscosity $\mu$. In this study, we distinguish the dynamic viscosity behind the upwind and downwind turbines, i.e., $\mu_{1 \rightarrow 3}=\mu_{2 \rightarrow 4}=0 \mathrm{~kg} / \mathrm{ms}$ and $\mu_{3 \rightarrow 5}=\mu_{4 \rightarrow 6}=10 \mathrm{~kg} / \mathrm{ms}$, to create different rate of wake recovery, in accordance with the simulated wind farm layout with PALM. Note that $\mu_{i \rightarrow j}$ is the dynamic viscosity between the $i^{t h}$ upwind turbine and the $j^{\text {th }}$ downwind turbine in the simulated wind farm model with WFSim.

In order to evaluate the dynamic performance of the WFSim model with the PALM as a reference, both models are excited using the same time-series of the axial induction factors. Figure 5 shows the time series of the applied induction factors, taken from [18], to the individual turbines of the wind farm models. The corresponding averaged rotor velocities are demonstrated for clear assessments of the used model. The wake-induced velocity deficits at downwind rotor locations are captured in an acceptable agreement with the large-eddy simulation model. Figure 6 shows that the total power production of the investigated model is well estimated, compared with the PALM total power production. 

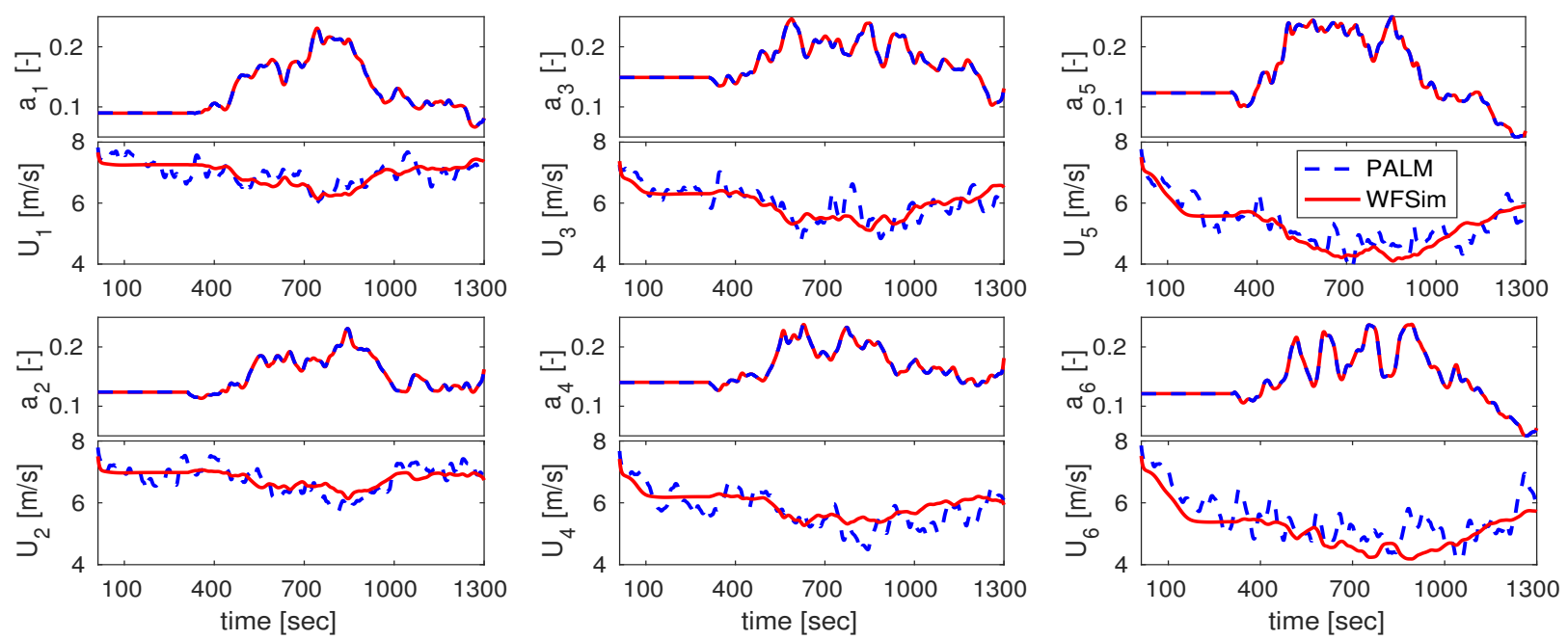

Figure 5: The applied axial induction factors and the corresponding estimated rotor-averaged flow velocities of the individual wind turbines, compared with those of the high-fidelity PALM model.

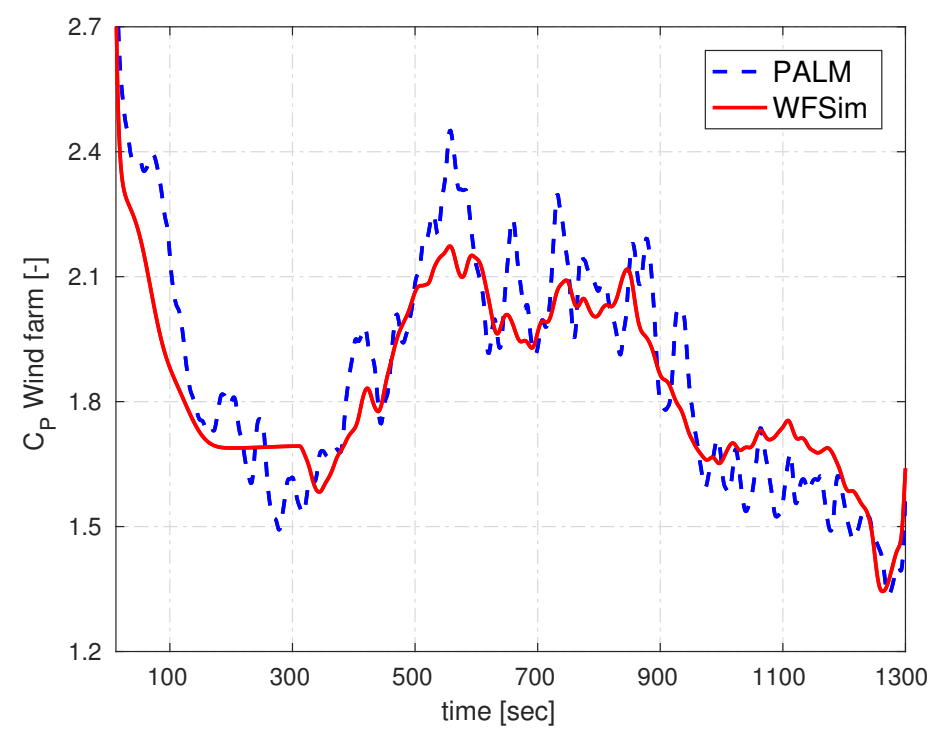

Figure 6: The total power coefficient of WFSim, compared with PALM for the simulated wind farm example. The power coefficient is computed as $C_{P_{W F}}=\sum_{i=1}^{N_{t}} C_{P_{i}}$ for the mean wind speed of $8 \mathrm{~m} / \mathrm{s}$.

\section{Results and discussion}

The performance of the adjoint-based model predictive controller (AMPC) is analyzed here through simulation studies with time-varying atmospheric conditions. The investigated layout (see Fig. 4) is simulated with the WFSim model with the key parameters given in Table B.3. We consider the performance of the controller in the below-rated region, wherein the wind speed is lower than its rated value. The main control objective here is to operate at the optimal point on a wind farm level, i.e., capturing the kinetic energy of the wind as much as possible. Although this optimal point is the same with respect to different wind speeds at this below-rated region, it varies with changes in the wind direction due to different wake trajectories, and therefore different wake interactions. Hence, the wind farm controller must be able to adjust the control inputs in such a way that the wind farm always operates at the corresponding optimal point.

First, we explore the main features of the designed controller which should be considered for power maximization 
of waked wind farms. Then, the AMPC is examined to adjust the optimal wind turbine induction factors while the wind horizon yields more losses in the transient period because a higher importance is given to the steady-state solution.

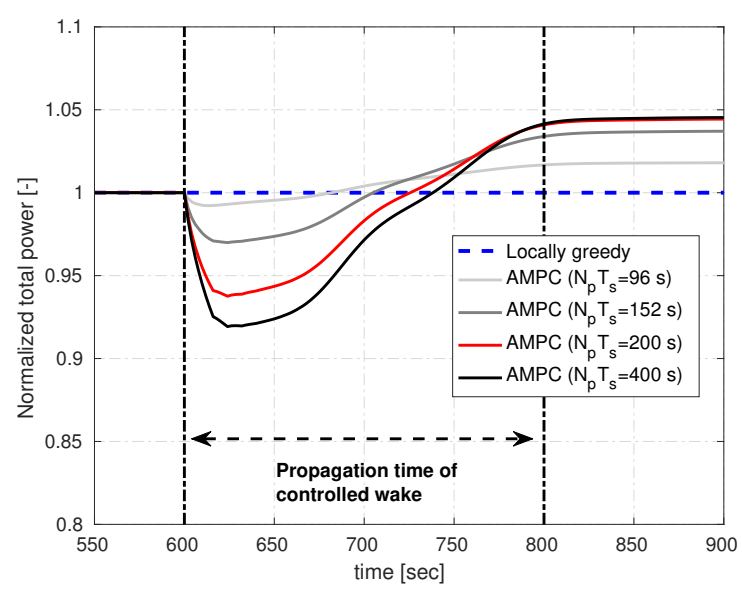

(a) Different lengths of the prediction horizon $N_{p}$ with $T_{s}=8 \mathrm{~s}$.

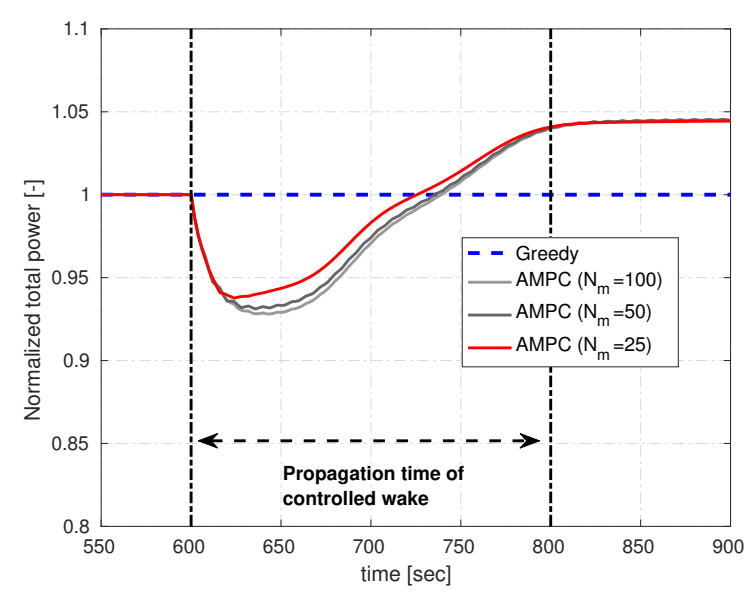

(b) Different numbers of moves $N_{m}$ with $N_{p} T_{s}=200 \mathrm{~s}$.

Figure 7: Normalized optimal control of energy extraction of the wind farm with different parameterization of the AMPC. The receding horizon length and the final time period are chosen as $N_{u} T_{s}=8 \mathrm{~s}$ and $\left\lfloor N_{p} / 2\right\rfloor$, respectively. The power is normalized with respect to the total power when each turbine is controlled to be locally greedy. 
The adjoint-based calculation of the gradient has efficiently the same computational cost as the employed model does for predicting dynamic flow and wake interactions over the prediction horizon. However, a reliable and feasible real-time wind farm control needs faster evaluations of the MPC performance index. The original AMPC problem [20] considers the full control DoFs, i.e., the number of wind turbine control inputs multiplied by the number of prediction samples. One possible solution to deal with the computational burden of the AMPC is to increase the time step of the controller, i.e., the control DoFs are effectively reduced. To this aim, a design parameter $N_{m}$ is introduced, defining the number of moves over the prediction horizon $N_{p}$, in order to reduce the number of performance index evaluations even more and consequently limit effectively the computational complexity of the AMPC.

Figure $7 \mathrm{~b}$ illustrates the optimal control of energy extraction with different numbers of moves $N_{m}$ over the selected prediction horizon $N_{p} T_{s}=200 \mathrm{~s}$. The selected number of moves $N_{m}=100$ represents the performance of the original full-order AMPC [20] for the conducted simulation set-up with the chosen sample time $\Delta t=2 \mathrm{~s}$. Indeed, reducing the time step of the MPC scheme might sacrifice the closed-loop performance in an undesirable manner particularly for systems with fast dynamics. The accuracy of the AMPC for optimal energy extraction remains almost unchanged for the reduced order AMPC, e.g., by limiting the number of moves to $N_{m}=25$. The detailed analysis of the computational complexity of the AMPC with the proposed parameterization is provided in section 6

\subsection{Steady-state optimal references}

To evaluate the performance of the AMPC with dynamic wake interactions, we first search for the steady-state optimal control settings for two different wind farm operating conditions, full and partial wake interactions. First, we assume that the inflow is aligned to the rotor discs and wind turbines interact fully through their wakes. Second, the incoming wind is misaligned $8^{\circ}$ with the rotor discs due to a wind direction change, that mitigates aerodynamic interactions of the wind turbines. Similar to [20], the flow is simulated for laminar flow conditions with constant viscosity parameter $\mu=10 \mathrm{~kg} / \mathrm{ms}$, where viscous forces are dominant. To find the optimal steady-state axial induction factors, we employed a Game Theoretic (GT) approach [10], an iterative open-loop control strategy, proposed for maximizing the power production of waked wind farms.

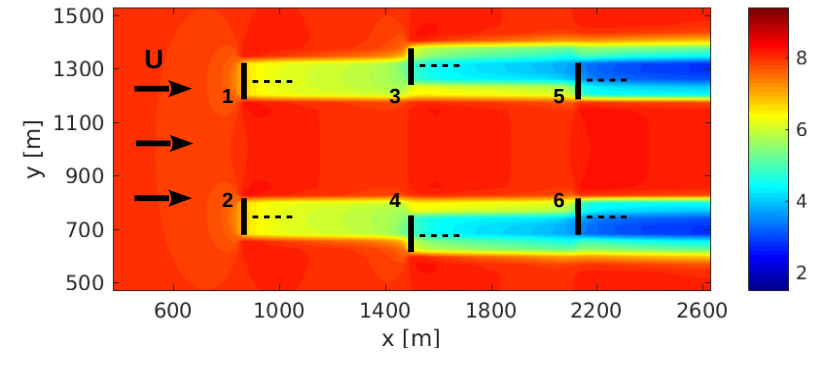

(a) Full wake interactions

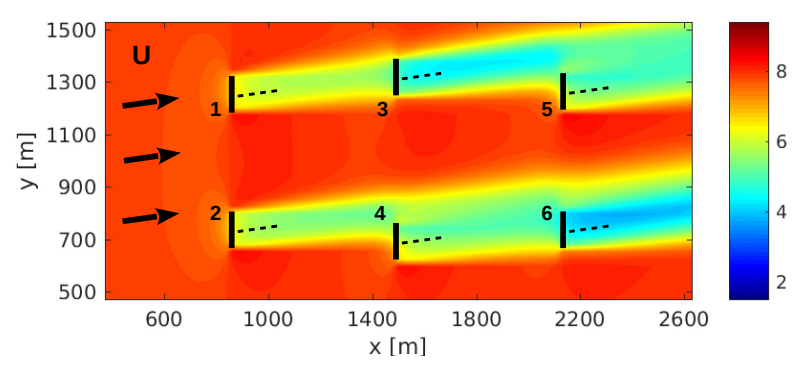

(b) Partial wake interactions

Figure 8: Six-turbine example operating with the optimal axial induction factors, achieved using GT approach, at $U_{\infty}=8 \mathrm{~m} / \mathrm{s}$. The incoming flow is aligned to the rotor discs (left) and misaligned $8^{\circ}$ with the rotor discs (right), resulting in full and partial wake interactions, respectively.

Figure 8 depicts our six-turbine example operating with the steady-state optimal axial induction factors for two different wind farm operating conditions. The corresponding optimal control settings, achieved by the GT approach, are listed in Table 1 for each case. It should be noted that the asymmetric coordination of the induction factors for the partial 
wake relates to different wake interactions at each row of turbines, caused by the $8^{\circ}$ wind direction change (see Fig. $8 \mathrm{~b}$ ). The steady-state simulation results with WFSim show that there exists the potential of a $4 \%$ power increase for the full wake conditions and a $1.5 \%$ increase for the partial wake conditions, with respect to the locally greedy control approach, via the optimal coordination of the wind turbine control settings. The lower power gain for the partial wake condition is related to the lower wake-induced power losses.

Table 1: Steady-state optimal set-points of wind turbines, achieved using GT approach, for two different wind directions.

\begin{tabular}{lllllll}
\hline & \multicolumn{3}{c}{ Full-wake $\left(0^{\circ}\right)$} & \multicolumn{3}{c}{ Partial-wake $\left(8^{\circ}\right)$} \\
\hline$a_{1}=0.21$ & $a_{3}=0.28$ & $a_{5}=0.32$ & $a_{1}=0.26$ & $a_{3}=0.31$ & $a_{5}=0.32$ \\
$a_{2}=0.21$ & $a_{4}=0.28$ & $a_{6}=0.32$ & $a_{2}=0.31$ & $a_{4}=0.26$ & $a_{6}=0.33$ \\
\hline
\end{tabular}

\subsection{Optimal control of energy extraction with dynamical changes in wind direction}

In order to evaluate the performance of the AMPC, the following simulation scenario is defined. The individual wind turbines start operating with the optimal axial induction factors, while the wind direction is aligned to the rotor discs (see Fig. 8a. After $600 \mathrm{~s}$, the wind direction at the boundary conditions at the west side of the domain is changed, yielding $8^{\circ}$ misalignment with the rotor discs and deflected wake propagations (see Fig. 8b). After 600 additional seconds, the wind turns again into the initial direction and as a result wake interactions change dynamically. The key parameters of the AMPC are chosen here as the prediction horizon $N_{p} T_{s}=200 \mathrm{~s}$, the receding horizon $N_{u} T_{s}=8 \mathrm{~s}$, the final time period $N_{f} T_{s}=96 \mathrm{~s}$, and the number of moves $N_{m}=25$. The AMPC step time and the simulation sample time are set to $T_{s}=8 \mathrm{~s}$ and $\Delta t=2 \mathrm{~s}$, respectively. Note that the length of the receding horizon $N_{u} T_{s}=8 \mathrm{~s}$ guarantees a satisfactory closed-loop wind farm performance with changes in wind direction while the computational complexity of the AMPC optimization problem is kept relatively low.

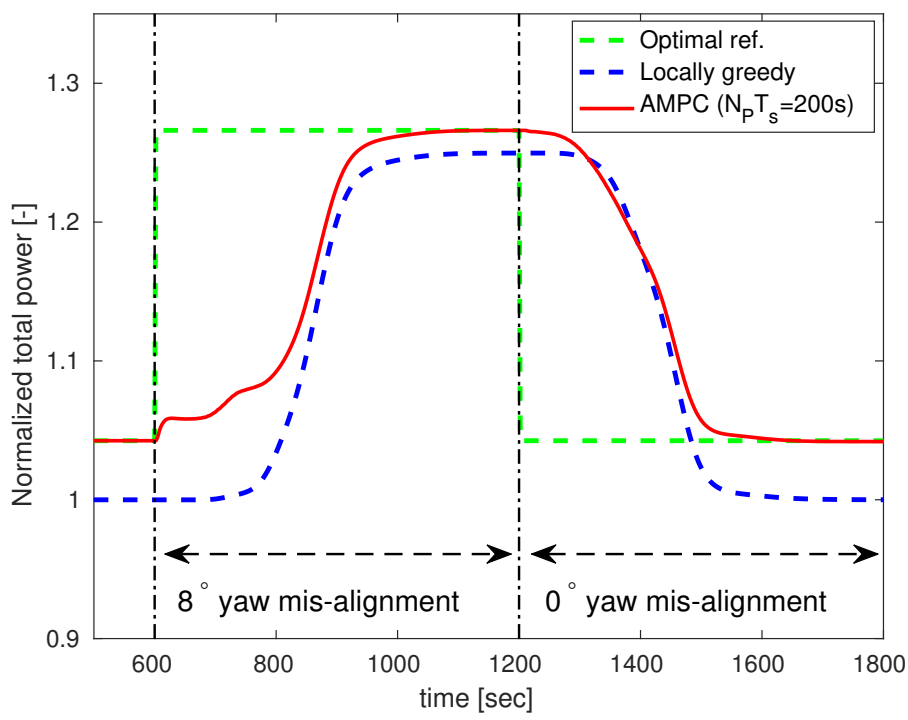

Figure 9: Normalized total power production with AMPC, while wind direction changes. The power is normalized with respect to the total power of the locally greedy control at the full-wake operating condition. Green dashed lines represent the steady-state maximum power set-points of the wind farm, obtained by the GT approach.

Figure 9 shows the optimal control of the energy extraction of our simulated example with wind direction changes, 
compared with the locally greedy control $\left(a_{i}=0.33\right)$. It can be seen that the AMPC is able to maximize the power production, while its optimal operating point is altered due to changes in the atmospheric conditions. The controller reacts relatively fast, because the control inputs and wind farm responses are predicted in advance and optimized with respect to the total power production. Note that the green dashed curve represents the steady-state maximum total power set-points, corresponding to Table 1 for each yaw mis-alignment. The total power production of the wind farm converges to the corresponding steady-state optimal reference after controlled wakes propagate within the wind farm.
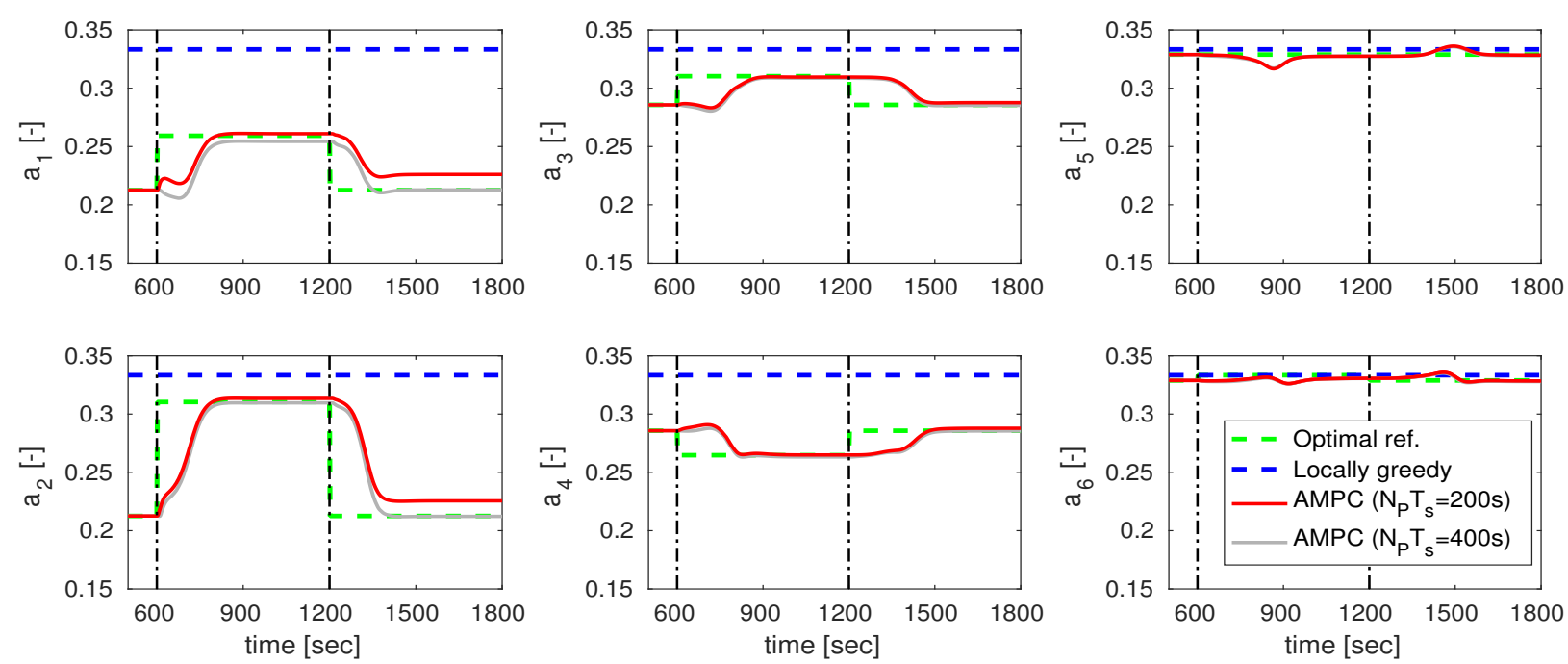

Figure 10: The induction factors of the individual wind turbines operating with AMPC, compared with the locally greedy control $a_{i}=0.33$. Green dashed lines represent the steady-state optimal set-points of the wind turbines, computed by the GT approach.

Figure 10 illustrates the time-varying behaviour of the axial induction factors of the individual wind turbines. The control input trajectories converge to the steady-state optimal solutions (green dashed lines), obtained by the GT approach (see Table 1, after wake traveling time. The observed delay in the convergence time corresponds to the flow and wake traveling times. Note that at time instants $600 \mathrm{~s}$ and $1200 \mathrm{~s}$, the wind direction is changed at the west boundaries of the wind farm domain. The induction factors of the upwind turbines are adjusted when new atmospheric conditions reach them, taking their impacts on the downwind turbines into account. The last machines, i.e., 5th and 6th turbines, are operating always with the greedy control setting to capture as much kinetic energy as possible from the incoming wind. In the partial wake condition, the 2nd and 3rd turbines are controlled to operate closer to greedy because the deflections of their wakes away from downwind turbines are predicted and taken into account (see Fig. 8b).

One important note here is that the prediction horizon $N_{p} T_{s}=200 \mathrm{~s}$ is chosen on the basis of the wake traveling time from an upwind turbine toward its first downwind one. It means that the wake impacts of the 1 st and 2 nd turbines on the 5th and 6th turbines, respectively, cannot be seen by the predictive controller, which results in a sub-optimal solution at the steady state condition, compared with the steady-state optimal set-points of the wind turbines, computed by the GT approach (see $a_{1}$ and $a_{2}$ of Fig. 10). As shown, the accuracy of the controller is improved by a selection of the longer prediction horizon $N_{p} T_{s}=400 \mathrm{~s}$ (see light grey curves), which is enough time for propagation of wakes within the given wind farm example. However, little amount of the total power gain compared with the shorter prediction horizon $N_{p} T_{s}=200 \mathrm{~s}$ is negligible with respect to the increased amount of computational effort.

Figure 11 illustrates the normalized power production of the individual wind turbines. When the inflow has $8^{\circ}$ misalignment with the rotor area, the 2nd and 5th turbines produce the same level of greedy control because their wakes 

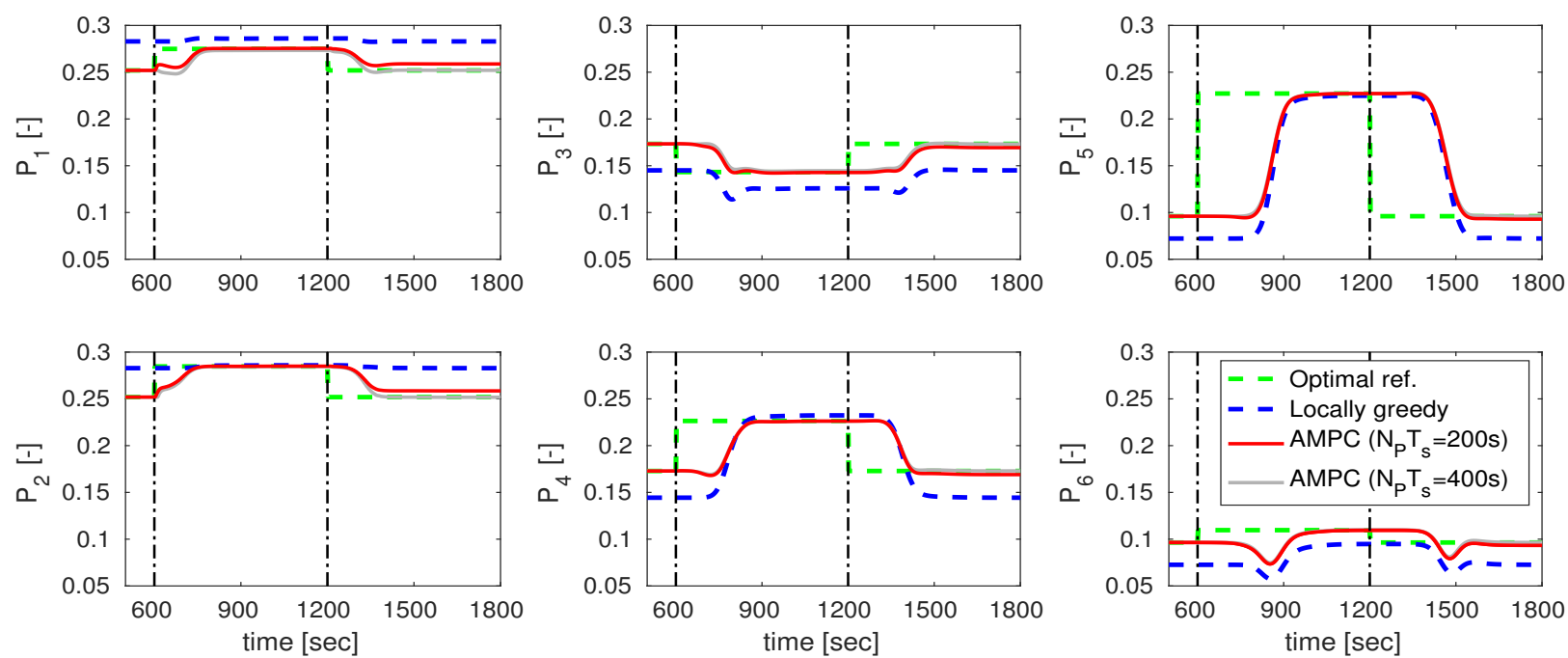

Figure 11: The normalized power of the individual wind turbines operating with AMPC, compared with the locally greedy control. The power is normalized with respect to the total power of the locally greedy control at the full-wake operating condition. Green dashed lines represent the steady-state optimal power set-points of the wind turbines, computed by the GT approach.

do not interact significantly with other turbines. The noticeable power increases of the 4 th and 5 th machines are due to the deflected wakes of their upwind turbines (see Fig. 8b). While the wind turbines are fully interacting through their wakes (last $10 \mathrm{~min}$ ), the controller reduces the energy extraction of the upwind turbines to increase the kinetic energy of

\section{Considerations for real-time implementation}

In order to consider the proposed AMPC for the real-time control implementations, several key factors should be taken into account for reducing the computational complexity of the controller. The analysis of the prediction horizon $N_{p}$, as a key parameter design of AMPC, is discussed in section 5.1. With a long prediction horizon, probable influences of an upwind turbine on all downwind turbines can be captured, which improves the accuracy of the controller at the 


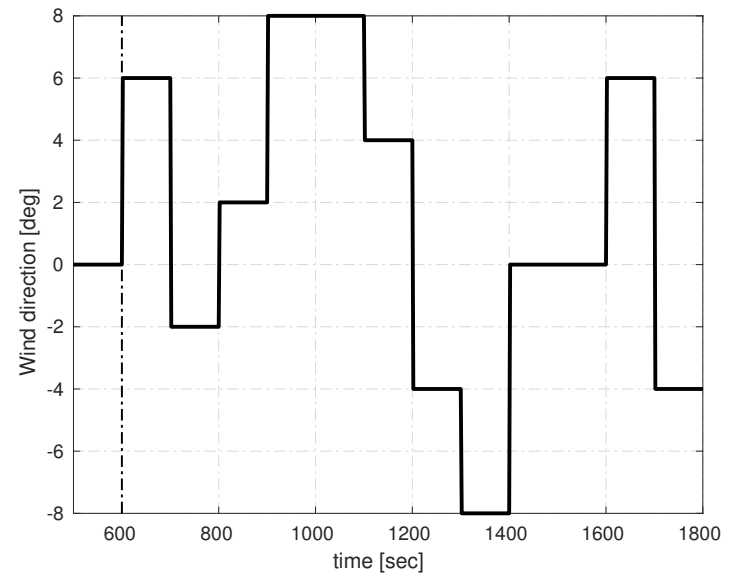

(a) Wind direction change

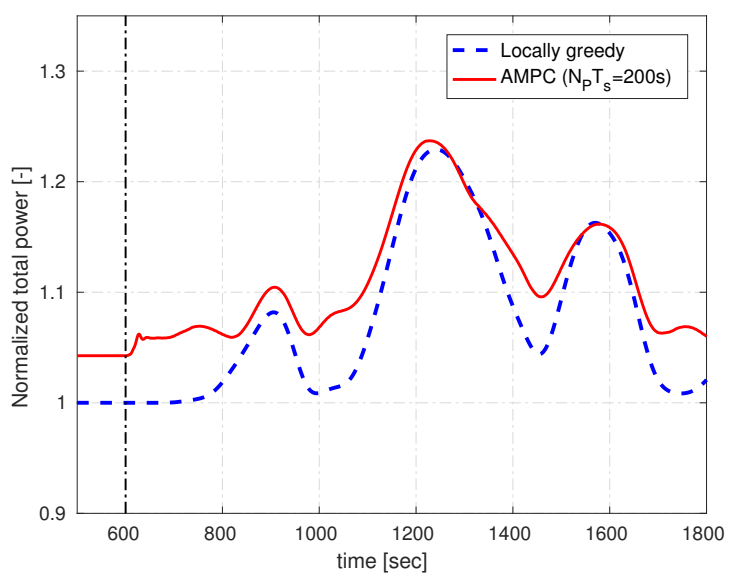

(b) Optimal control of energy extraction

Figure 12: Normalized total power production with AMPC, while the wind direction changes quickly. The power is normalized with respect to the total power of the locally greedy control at the full-wake operating condition.
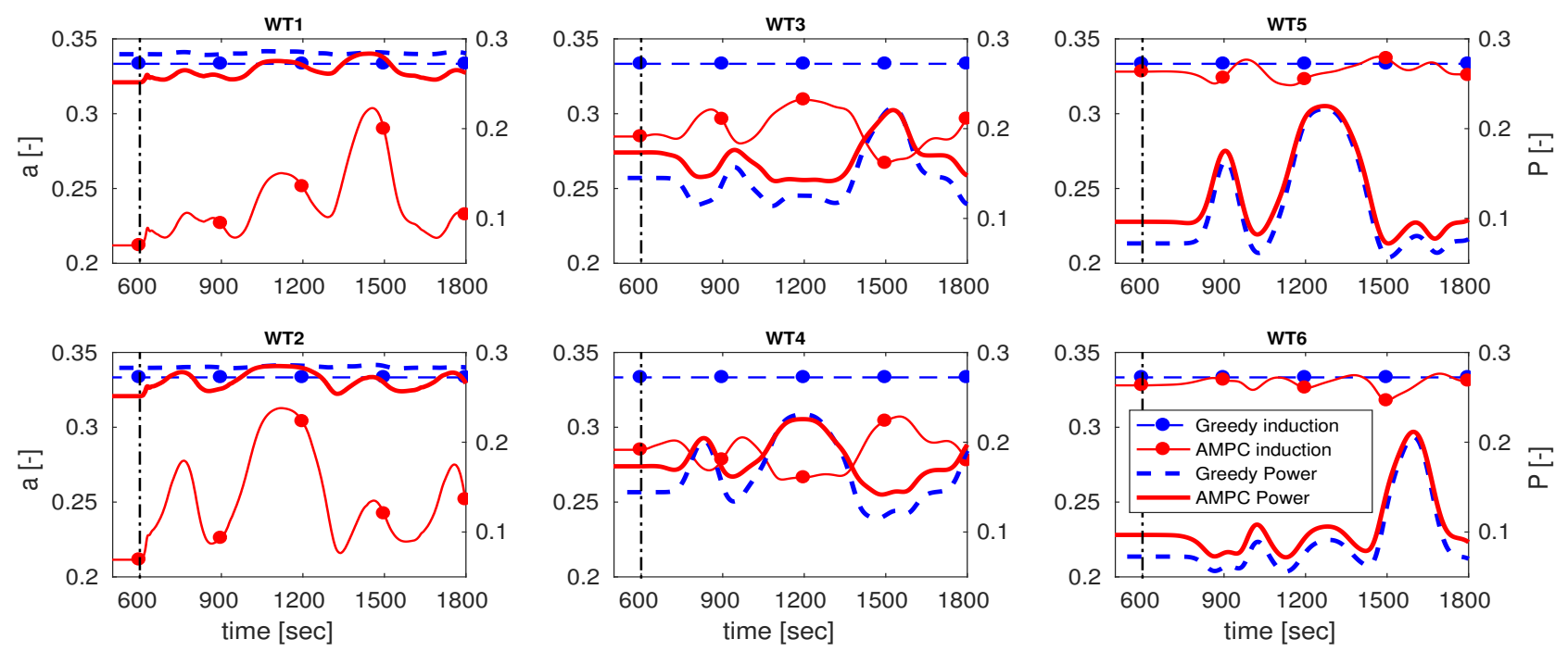

Figure 13: The axial induction factor trajectories and the normalized power of the individual wind turbines operating with AMPC, compared with the locally greedy control. The left and right vertical axes represent the induction factor and the normalized power, respectively. The powers are normalized with respect to the total power of the locally greedy control at the full-wake operating condition.

expense of additional computation, as shown in Fig. 10 This section summarizes other major elements that influence the computational complexity of AMPC, which correspond to the systematic procedure of the AMPC implementation shown in Fig. 3 .

In section 5.1, reducing the control DoF of AMPC is discussed by limiting the number of moves $N_{m}$ over the prediction horizon, which yields fewer numbers of linearization and performance index evaluation. As discussed, the adjointbased calculation of the gradient has almost the same computational cost as the employed model does, regardless of the number of the decision variables. Therefore, the complexity of the model, which is determined by the chosen domain size and the defined staggered grid resolution, is another key factor specifying the computational complexity of AMPC. The computational efficeincy of the WFSim is clear enough, compared with an LES model (see Table B.3). However, the employed model of the investigated $2 \times 3$ wind farm example is described with $n=19824$ state variables, containing veloc- 
ity and pressure of all grid points, for predicting dynamic flow and wake interactions among wind turbines. Therefore, further study on the model reduction is also needed for the fast and reliable performance of AMPC.

In the following, a comparative study is conducted to evaluate the influence of the aforementioned factors on both the computational burden and the performance quality of the AMPC for optimal energy extraction of the given wind farm example. The simulation scenario with multiple changes in wind direction is used with sample time $\Delta t=2 \mathrm{~s}$. The prediction horizon and the receding horizon are chosen as $N_{p} T_{s}=200 \mathrm{~s}$ and $N_{u} T_{s}=8 \mathrm{~s}$, respectively for all cases. The original AMPC [20] is considered as a reference with full-order optimization domain, i.e., the sample time of the controller model and the simulation model is equal $\left(T_{s}=\Delta t\right)$. Furthermore, two different coarse grids are generated for WFSim, which result in reduced order models for prediction of wind farm responses of the same domain. Note that the parameterization of the wind farm simulation model is kept the same as before for all case studies. Table 2 summarizes the different parameterization of the AMPC, involving the specified model order and the number of moves. The detailed elapsed time for each computing steps and a receding horizon control are outlined to assess the impact of AMPC parameterization on its computational complexity. Since the number of iterations is variable, it is limited to two iterations per each control action for a better comparison of the controllers performance. For this study, the simulations were run on the EDDY HPC (High-Performance Computing) cluster [42] with a single core.

Table 2: Evaluation of computational complexity and control performance with different parametrizations of the AMPC. The lengths of the prediction horizon and receding horizon are chosen as $N_{p} T_{s}=200 \mathrm{~s}$ and $N_{u} T_{s}=8 \mathrm{~s}$, respectively.

\begin{tabular}{|c|c|c|c|c|c|}
\hline AMPC parameterization & $\begin{array}{l}\text { Number of grid cells } \\
\text { WFSim model order } n \\
\text { Turbine grid cells No. } \\
\text { Number of moves } N_{m} \\
\text { AMPC time step } T_{s}\end{array}$ & $\begin{array}{c}{[100 \times 70]} \\
19824 \\
5 \\
100^{1} \\
2 \mathrm{~s} \\
\end{array}$ & $\begin{array}{c}{[100 \times 70]} \\
19824 \\
5 \\
25 \\
8 \mathrm{~s} \\
\end{array}$ & $\begin{array}{c}{[60 \times 44]} \\
7206 \\
4 \\
25 \\
8 \mathrm{~s} \\
\end{array}$ & $\begin{array}{c}{[50 \times 31]} \\
4097 \\
3 \\
25 \\
8 \mathrm{~s} \\
\end{array}$ \\
\hline $\begin{array}{l}\text { Elapsed time } T_{1} \\
\text { (Linearization and storage) }\end{array}$ & & $72.63 \mathrm{~s}$ & $15.48 \mathrm{~s}$ & $3.79 \mathrm{~s}$ & $2.01 \mathrm{~s}$ \\
\hline $\begin{array}{l}\text { Elapsed time } T_{2} \\
\text { (Adjoint-based gradient calculation) }\end{array}$ & & $15.68 \mathrm{~s}$ & $4.28 \mathrm{~s}$ & $1.46 \mathrm{~s}$ & $0.84 \mathrm{~s}$ \\
\hline $\begin{array}{l}\text { Elapsed time } T_{3} \\
\text { (Forward prediction) }\end{array}$ & & $13.51 \mathrm{~s}$ & $3.70 \mathrm{~s}$ & $1.20 \mathrm{~s}$ & $0.65 \mathrm{~s}$ \\
\hline $\begin{array}{l}\text { Total elapsed time } T \\
\text { (One receding horizon control) }\end{array}$ & & $216.7 \mathrm{~s}$ & $47.1 \mathrm{~s}$ & $12.8 \mathrm{~s}$ & $7.0 \mathrm{~s}$ \\
\hline $\begin{array}{l}\text { Changes of computational complexity } \\
\text { (w.r.t. full order AMPC [20]) }\end{array}$ & & NA & $-78.3 \%$ & $-94.1 \%$ & $-96.8 \%$ \\
\hline $\begin{array}{l}\text { Wind farm power gain } \\
\text { (w.r.t. locally greedy control) }\end{array}$ & & $+3.24 \%$ & $+3.24 \%$ & $+3.15 \%$ & $+3.12 \%$ \\
\hline
\end{tabular}

${ }^{1}$ Full order AMPC optimization problem as the reference [20]

${ }^{2}$ Sum of the total power gain over 1200 s simulation run with AMPC

For all cases, the dominant computing effort corresponds to the first step involving the linearization of the wind farm flow model and the performance index over the prediction horizon. Reducing the number of decision variables limits significantly the elapsed time for all computing steps. As expected, the adjoint-based gradient is calculated almost with the same computational cost of the forward prediction, i.e, $T_{2} \approx T_{3}$. Note that the adjoint propagates backward in time using the family of linearized models, which are stored in the first step, and associates the variations of the model and the performance index. The impact of the employed model order on the computational complexity of AMPC is also obvious.

The performance of each controller parameterization is assessed using the power gains of the wind farm example with 
respect to the locally greedy control. Figure 14 plots the optimal control of energy extraction with frequent changes in wind direction for all studied parameterizations. As reported in Table 2 , the optimal energy extraction of the wind farm is slightly reduced as a result of limiting the complexity of the AMPC wind farm model. Comparing with the full order AMPC optimization problem with $N_{m}=100$ [20], the execution time is significantly reduced (approximately 78\%) due to fewer evaluations of the performance index with $N_{m}=25$. Indeed, the longer MPC time step $T_{s}=8 \mathrm{~s}$ is able to capture the dominant wake dynamics needed for the closed-loop control of waked wind farms. The total elapsed time $T$ falls below the chosen receding horizon control $N_{u} T_{s}=8 \mathrm{~s}$ for WFSim parameterization with the order of $n=4097$. Further improvements can be expected by, e.g., investigating the model order reduction and the code optimization. The future work focuses on the implementation of the AMPC in PAarallelize Large-eddy Simulation Model of wind farms in order to examine its performance under detailed dynamic wake and turbulence conditions of wind farm flows.

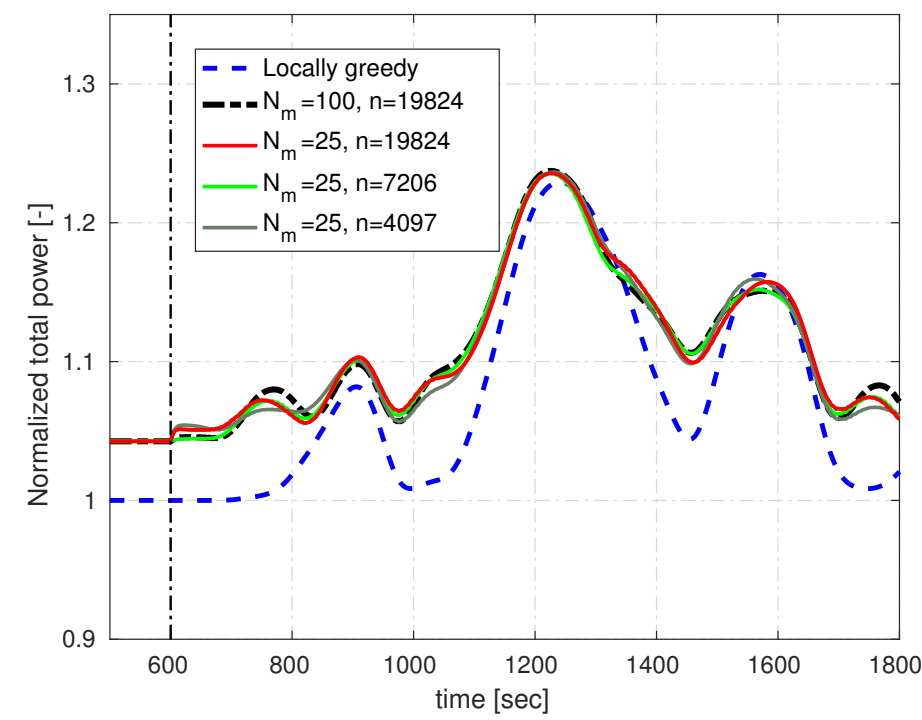

Figure 14: Normalized total power production of wind farm with different parameterization of AMPC according to Table 2 for analyzing its computational burden. The power is normalized with respect to the total power of the locally greedy control at the full-wake operating condition. $n$ represents the order of the AMPC model and $N_{m}$ stands for the number of moves over the prediction horizon $N_{p} T_{s}=200 \mathrm{~s}$.

\section{Conclusion}

An adjoint-based model predictive control (AMPC) has been proposed in this study for optimal energy extraction of wind farms, considering the aerodynamic wake interactions among wind turbines. The MPC optimization problem is formulated to maximize the total power production over a prediction horizon, subjected to a 2D dynamic wind farm model, the so-called WFSim. The developed controller benefits from an adjoint approach as a computationally-efficient tool for computing the gradient. The employed WFSim model is validated with a LES wind farm model in order to demonstrate its capability of capturing the dominant dynamics of the wake interaction at hub height of the wind turbines. The key design parameters of the developed AMPC are elaborately discussed for a practically optimal energy extraction in a waked wind farm. The length of the prediction horizon should be long enough to capture the propagation of a wake and to limit the undesirable effects caused by the finite-horizon optimal control simultaneously. The computational complexity of the AMPC is limited effectively by increasing the time steps of the controller. 
The effectiveness of the proposed approach is investigated using an example $2 \times 3$ wind farm. The wake challenge is first elaborated to demonstrate the wake-induced power losses and the dependency of the wake interactions on changes in atmospheric conditions. Contrary to open-loop control strategies, based on quasi steady-state inflow conditions, the AMPC is capable of reacting to varying atmospheric and operational conditions using feedback. Simulation results demonstrate that the AMPC converges reasonably quick to the optimal control set-points, while wake interactions are perturbed with changes in wind direction. In comparison to the conventional locally greedy control, the AMPC yields higher power gain, i.e. up to $4 \%$ increase, when the wind turbines operate with full wake interactions. It is also demonstrated that an appropriate parameterization of the AMPC can lead to a significant reduction of the execution time without degrading the control performance. Although the obtained $7 \mathrm{~s}$ executation time is a promising result, further study of the model reduction and the code optimization can still be done. The implementation of the developed control approach in PALM is our current focus to assess the AMPC performance and realization, under detailed dynamic wake and turbulence conditions in a simulated wind farm.

\section{Acknowledgement}

The main author especially wants to thank Gerald Steinfeld and Lukas Vollmer for their kind support with PALM simulations.

This work has been funded by the German Ministry of Economic Affairs and Energy (BMWi) in the scope of the "WIMS-Cluster" project (FKZ 0324005) and by the Ministry for Science and Culture of Lower Saxony through the funding initiave "Niedersächsisches Vorab" in the scope of the "ventus efficiens" (ZN3024). Support from a fellowship from the Hanse-Wissenschaftskolleg in Delmenhorst, Germany is also gratefully acknowledged.

\section{Appendix A. Partial derivatives of the wind farm model over one prediction horizon}

The derivatives of the discretized wind farm model $\tilde{\mathbf{C}}(\tilde{X}, \tilde{\boldsymbol{\beta}})=0$ with respect to the state $\tilde{X}$ and control input $\tilde{\boldsymbol{\beta}}$, over the whole prediction horizon $N_{p}$, are

$$
\begin{aligned}
& \tilde{\mathbf{C}}_{\tilde{X}}=\left[\begin{array}{ccccc}
\left(C_{1}\right)_{X_{1}} & 0 & \cdots & 0 & 0 \\
\left(C_{2}\right)_{X_{1}} & \left(C_{2}\right)_{X_{2}} & \cdots & 0 & 0 \\
\vdots & \ddots & \ddots & \vdots & \vdots \\
0 & 0 & \ddots & \left(C_{N_{p}-1}\right)_{X_{N_{p}-1}} & 0 \\
0 & 0 & \cdots & \left(C_{N_{p}}\right)_{X_{N_{p}-1}} & \left(C_{N_{p}}\right)_{X_{N_{p}}}
\end{array}\right] \\
& \tilde{\mathbf{C}}_{\tilde{\beta}}=\left[\begin{array}{cccccc}
0 & 0 & \cdots & 0 & 0 & 0 \\
\left(C_{2}\right)_{\beta_{1}} & 0 & \cdots & 0 & 0 & 0 \\
0 & \left(C_{3}\right)_{\beta_{2}} & \cdots & 0 & 0 & 0 \\
\vdots & \vdots & \ddots & \vdots & \vdots & \vdots \\
0 & 0 & \cdots & \left(C_{N_{p}-1}\right)_{\beta_{N_{p}-2}} & 0 & 0 \\
0 & 0 & \cdots & 0 & \left(C_{N_{p}}\right)_{\beta_{N_{p}-1}} & 0
\end{array}\right]
\end{aligned}
$$


where the linearized components of the discretized wind farm model $[15]$ at sample time $k$ are

$$
\begin{aligned}
\left(C_{k}\right)_{X_{k}} & =\boldsymbol{E}\left(X_{k-1}\right) \\
\left(C_{k}\right)_{X_{k-1}} & =\left(\boldsymbol{E}\left(X_{k-1}\right) X_{k}\right)_{X_{k-1}}-\boldsymbol{A}-\left(\boldsymbol{B}\left(X_{k-1}\right) \boldsymbol{\beta}_{k-1}\right)_{X_{k-1}}-\left(b\left(X_{k-1}\right)\right)_{X_{k-1}}, \\
\left(C_{k}\right)_{\beta_{k-1}} & =-\boldsymbol{B}\left(X_{k-1}\right) .
\end{aligned}
$$

The reader is referred to [25] for more details on the linearized model.

\section{Appendix B. Simulation set-ups for wind farm model validation}

Table B.3 summarizes the key parameters of the simulation set-ups used for both the WFSim and PALM. Figure B.15 plots the instantaneous field of the $u$-components of the wind at hub-height of the wind turbines simulated with both the considered models.

Table B.3: The key parameters of the WFSim and PALM simulation set-ups.

\begin{tabular}{lcc}
\hline Simulation set-up & WFSim (2D) & PALM (3D) \\
\hline Domain size $L_{x} \times L_{y} \times L_{z}$ & $3 \times 2 \times[-] \mathrm{km}^{2}$ & $15.3 \times 3.8 \times 1.3 \mathrm{~km}^{3}$ \\
Grid mesh size $N_{x} \times N_{y} \times N_{z}$ & $100 \times 70 \times[-]$ & $1024 \times 256 \times 128$ \\
Cell mesh resolusion $\Delta_{x} \times \Delta_{y} \times \Delta_{z}$ & $30 \times 29 \times[-] \mathrm{m}^{2}$ & $15 \times 15 \times 10 \mathrm{~m}^{3}$ \\
Number of wind turbines & 6 & 6 \\
Wind turbine model & corrected ADM & $\mathrm{ADM}^{1}$ \\
Wind turbine control DoF & Induction factor & Induction factor \\
Turbine rotor diameter $D$ & $126 \mathrm{~m}$ & $126 \mathrm{~m}$ \\
Number of grid cells per turbine & 5 & 68 \\
Hub height & {$[-]$} & $90 \mathrm{~m}$ \\
Atmospheric stability condition & {$[-]$} & $\mathrm{NBL}$ \\
Effective wind speed at hub height & $8 \mathrm{~m} / \mathrm{s}$ & $8 \mathrm{~m} / \mathrm{s}$ \\
Geostrophic wind velocity & {$[-]$} & $u=9 \mathrm{~m} / \mathrm{s} \mathrm{and} v=-2 \mathrm{~m} / \mathrm{s}$ \\
Sample time $\Delta t$ & $1 \mathrm{~s}$ & $1 \mathrm{~s}$ \\
Computation time & seconds & hours \\
\hline
\end{tabular}

${ }^{1}$ Actuator disc model

2 Neutral boundary layer

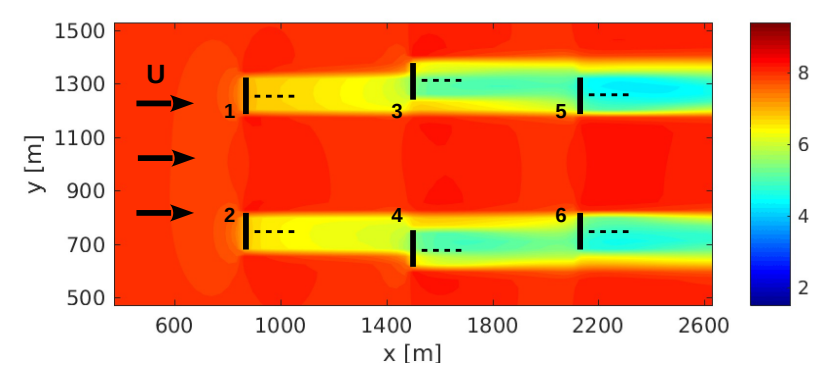

(a) WFSim model

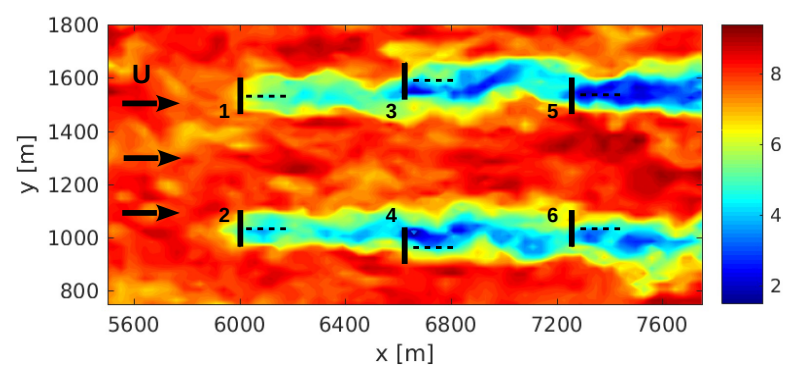

(b) PALM model horizontal snapshot at hub hight

Figure B.15: The simulated $2 \times 3$ wind farm flow with WFSim and PALM, taken from one random frozen time. 


\section{Appendix C. Characteristic performance of wind turbines}

The steady-state performance of the wind turbines with an undisturbed flow at ambient wind speed $8 \mathrm{~m} / \mathrm{s}$ are observed for both models. The unperturbed thrust and power coefficients of the wind turbines versus the axial induction factors are extracted as follows

$$
\begin{aligned}
& C_{T}(a)=\frac{T(a)}{\frac{1}{2} \rho A_{d} U_{\infty}^{2}}=\frac{4 a}{1-a}\left(\frac{C_{f}(a) U_{d}}{U_{\infty}}\right)^{2}, \\
& C_{P}(a)=\frac{P(a)}{\frac{1}{2} \rho A_{d} U_{\infty}^{3}}=\frac{4 a}{1-a}\left(\frac{C_{f}(a) U_{d}}{U_{\infty}}\right)^{3},
\end{aligned}
$$

in order to associate the performance of a single turbine incorporated in the 2D and 3D flow models of wind farms.

Figure C.16 demonstrates the undisturbed thrust and power coefficients of the wind turbine models in both WFSim and PALM, compared with the actuator disc theory. For the PALM model, a few 600-second simulations are conducted for varying axial induction factors without the correction factor, i.e., $C_{f}(a)=1$. For the WFSim, the thrust and power of the effective wind speed $U_{\infty}$ are computed at the horizontal plane, on the basis of (??)-(??), facing only the width of the 5 grid cells which cover the rotor disc $(5 \Delta y \approx D)$. The necessity of the introduced correction factor $C_{f}(a)$ in 77 for satisfying both actuator disc theory and PALM is attributed to the two-dimensional inflow of the WFSim model.

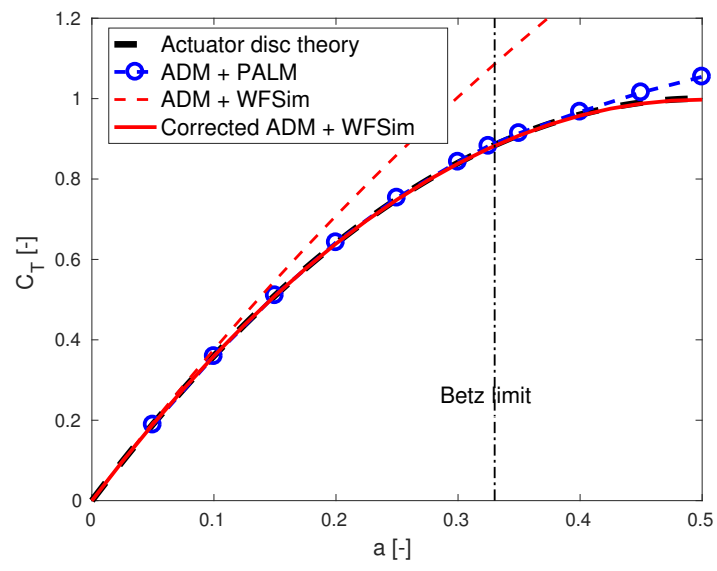

(a) Thrust coefficient

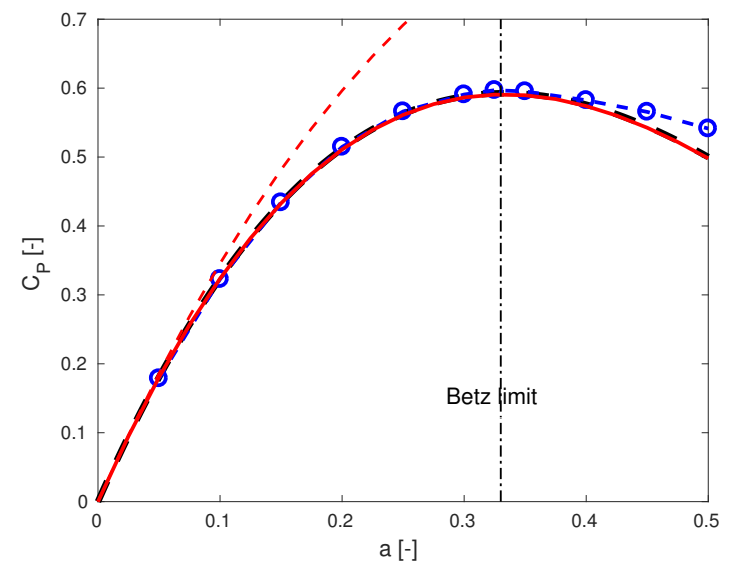

(b) Power coefficient

Figure C.16: Undisturbed thrust (a) and power (b) coefficients of the wind turbine models in both PALM and WFSim for the given wind farm, compared with actuator disc theory. The Betz limit referes to the operating point with maximum energy extraction of an isolated turbine.

\section{References}

[1] R. J. Barthelmie, K. Hansen, S. T. Frandsen, O. Rathmann, J. G. Schepers, W. Schlez, J. Phillips, K. Rados, A. Zervos, E. S. Politis, P. K. Chaviaropoulos, Modelling and measuring flow and wind turbine wakes in large wind farms offshore, Wind Energy 12 (5) (2009) 431-444. doi:10.1002/we.348

[2] T. Knudsen, T. Bak, M. Svenstrup, Survey of wind farm control: power and fatigue optimization, Wind Energy 18 (8) (2015) 1333-1351. doi:10.1002/we.1760. 
[3] S. Boersma, B. M. Doekemeijer, P. M. O. Gebraad, P. A. Fleming, J. Annoni, A. K. Scholbrock, J. A. Frederik, J. W. van Wingerden, A tutorial on control-oriented modeling and control of wind farms, in: American Control Conference, 2017, pp. 1-18. doi:10.23919/ACC.2017.7962923.

[4] J. Annoni, P. M. O. Gebraad, A. K. Scholbrock, P. A. Fleming, J.-W. van Wingerden, Analysis of axial-inductionbased wind plant control using an engineering and a high-order wind plant model, Wind Energy 19 (6) (2016) 1135-1150, we.1891. doi:10.1002/we.1891

[5] P. M. O. Gebraad, F. W. Teeuwisse, J. W. van Wingerden, P. A. Fleming, S. D. Ruben, J. R. Marden, L. Y. Pao, Wind plant power optimization through yaw control using a parametric model for wake effects: a CFD simulation study, Wind Energy 19 (1) (2016) 95-114, we.1822. doi:10.1002/we.1822.

[6] P. Fleming, J. Annoni, J. J. Shah, L. Wang, S. Ananthan, Z. Zhang, K. Hutchings, P. Wang, W. Chen, L. Chen, Field test of wake steering at an offshore wind farm, Wind Energy Science 2 (1) (2017) 229-239. doi:10.5194/ wes-2-229-2017

[7] M. Bromm, A. Rott, H. Beck, L. Vollmer, G. Steinfeld, M. Kühn, Field investigation on the influence of yaw misalignment on the propagation of wind turbine wakes, Wind Energy (in press) doi:10.1002/we.2210.

[8] F. Campagnolo, V. Petrović, C. L. Bottasso, A. Croce, Wind tunnel testing of wake control strategies, in: American Control Conference, 2016, pp. 513-518. doi:10.1109/ACC.2016.7524965.

[9] L. Vollmer, G. Steinfeld, D. Heinemann, M. Kühn, Estimating the wake deflection downstream of a wind turbine in different atmospheric stabilities: an LES study, Wind Energy Science 1 (2) (2016) 129-141. doi:10.5194/ wes $-1-129-2016$

[10] J. R. Marden, S. D. Ruben, L. Y. Pao, A model-free approach to wind farm control using game theoretic methods, IEEE Transactions on Control Systems Technology 21 (4) (2013) 1207-1214. doi:10.1109/TCST.2013. 2257780 .

[11] P. M. O. Gebraad, J. W. van Wingerden, Maximum power-point tracking control for wind farms, Wind Energy 18 (3) (2015) 429-447. doi:10.1002/we.1706

[12] U. Ciri, M. Rotea, C. Santoni, S. Leonardi, Large-eddy simulations with extremum-seeking control for individual wind turbine power optimization, Wind Energy 20 (9) (2017) 1617-1634, we.2112. doi:10.1002/we.2112.

[13] F. Campagnolo, V. Petrović, J. Schreiber, E. M. Nanos, A. Croce, C. L. Bottasso, Wind tunnel testing of a closedloop wake deflection controller for wind farm power maximization, Journal of Physics: Conference Series 753 (3) (2016) 032006. doi:10.1088/1742-6596/753/3/032006.

[14] M. Soleimanzadeh, R. Wisniewski, K. Johnson, A distributed optimization framework for wind farms, Journal of Wind Engineering and Industrial Aerodynamics 123 (2013) 88 - 98. doi:http://dx.doi.org/10.1016/ j.jweia.2013.08.011.

[15] J. P. Goit, J. Meyers, Optimal control of energy extraction in wind-farm boundary layers, Journal of Fluid Mechanics 768 (2015) 5-50. doi:10.1017/jfm.2015.70 
[16] J. Annoni, P. Seiler, A low-order model for wind farm control, in: American Control Conference, 2015, pp. 17211727. doi:10.1109/ACC.2015.7170981.

[20] M. Vali, V. Petrović, S. Boersma, J.-W. van Wingerden, M. Kühn, Adjoint-based model predictive control of wind

[21] M. Vali, V. Petrović, S. Boersma, J. W. van Wingerden, L. Y. Pao, M. Kühn, Model predictive active power control of waked wind farms, in: Annual American Control Conference (ACC), 2018, pp. 707-714. doi:10.23919/ ACC.2018.8431391

[22] S. Boersma, M. Vali, M. Kühn, J. W. van Wingerden, Quasi linear parameter varying modeling for wind farm control using the 2D Navier-Stokes equations, in: American Control Conference, 2016, pp. 4409-4414. doi: $10.1109 / \mathrm{ACC} .2016 .7525616$

[23] B. M. Doekemeijer, J. W. van Wingerden, S. Boersma, L. Y. Pao, Enhanced Kalman filtering for a 2D CFD NS wind farm flow model, Journal of Physics: Conference Series 753 (5) (2016) 052015. doi:10.1088/1742-6596/ $753 / 5 / 052015$

[24] B. Witha, G. Steinfeld, M. Dörenkämper, D. Heinemann, Large-eddy simulation of multiple wakes in offshore wind farms, Journal of Physics: Conference Series 555 (1) (2014) 012108. doi:10.1088/1742-6596/555/1/ 012108

[25] S. Boersma, P. Gebraad, M. Vali, B. Doekemeijer, J. W. van Wingerden, A control-oriented dynamic wind farm flow 口 model: WFSim, Journal of Physics: Conference Series 753 (3) (2016) 032005. doi:10.1088/1742-6596/

[26] R. Gasch, J. Twele, Wind power plants: fundamentals, design, construction and operation, Springer Science \& Business Media, 2011.

[27] T. Knudsen, T. Bak, Simple model for describing and estimating wind turbine dynamic inflow, in: American Control Conference, 2013, pp. 640-646. doi:10.1109/ACC.2013.6579909.

[28] H. K. Versteeg, W. Malalasekera, An introduction to computational fluid dynamics: the finite volume method, Pearson Education, 2007. 
[29] B. Doekemeijer, S. Boersma, L. Pao, T. Knudsen, J.-W. van Wingerden, Online model calibration for a simplified les model in pursuit of real-time closed-loop wind farm control, Wind Energy Science Discussions 2018 (2018) 1-30. doi:10.5194/wes-2018-33.

[38] S. Qin, T. A. Badgwell, A survey of industrial model predictive control technology, Control Engineering Practice 11 (7) (2003) 733 - 764. doi:https://doi.org/10.1016/S0967-0661(02)00186-7

[39] R. Cagienard, P. Grieder, E. Kerrigan, M. Morari, Move blocking strategies in receding horizon control, Journal

[40] B. Maronga, M. Gryschka, R. Heinze, F. Hoffmann, F. Kanani-Sühring, M. Keck, K. Ketelsen, M. O. Letzel, M. Sühring, S. Raasch, The Parallelized Large-Eddy Simulation Model (PALM) version 4.0 for atmospheric and oceanic flows: model formulation, recent developments, and future perspectives, Geoscientific Model Development 8 (8) (2015) 2515-2551. doi:10.5194/gmd-8-2515-2015.

[41] J. M. Jonkman, M. L. Buhl, Fast manual user's guide, NREL report No. NREL/EL-500-38230, 2005.

[42] (2016). [link]

URL WWw.uni-oldenburg.de/fk5/wr/hochleistungsrechnen/hpc-facilities/eddy/ 